\title{
ITERATED INTEGRALS, DIAGONAL CYCLES AND RATIONAL POINTS ON ELLIPTIC CURVES
}

\author{
by
}

\author{
Henri Darmon, Victor Rotger \& Ignacio Sols
}

\begin{abstract}
The theme of this article is the connection between the pro-unipotent fundamental group $\pi_{1}(X ; o)$ of a pointed algebraic curve $X$, algebraic cycles, iterated integrals, and special values of $L$-functions. The extension of mixed Hodge structures arising in the second stage in the lower central series of $\pi_{1}(X ; o)$ gives rise to a supply of complex points on the Jacobian $\operatorname{Jac}(X)$ of $X$ indexed by Hodge cycles on $X \times X$. The main results of this note relate these points to the Abel-Jacobi image of Gross-Kudla-Schoen's modified diagonal in $X^{3}$, and express this Abel-Jacobi image in terms of iterated integrals. The resulting formula is the basis for the practical complex-analytic calculations of these points when $X$ is a modular (or Shimura) curve, a setting where the recent work [YZZ] of X. Yuan, S. Zhang and W. Zhang relates their non-triviality to special values of certain $L$-series attached to modular forms.

Résumé. - Le thème de cet article est le groupe fondamental pro-unipotent $\pi_{1}(X ; o)$ d'une courbe algébrique $X$ munie d'un point base et ses relations avec les cycles algébriques, les intégrales itérées, et les valeurs spéciales des fonctions $L$. Les extensions de structures de Hodge mixtes qui apparaissent dans le quotient de $\pi_{1}(X, o)$ par le deuxième terme de sa série centrale descendante donnent lieu à des points complexes de la Jacobienne $\operatorname{Jac}(X)$ de $X$. Ces points sont paramétrés par les cycles de Hodge de $X \times X$. On les relie aux images de la diagonale modifiée de Gross-Kudla-Schoen dans $X^{3}$ par l'application d'Abel-Jacobi, en exprimant ces images au moyen d'intégrales itérées. La formule explicite qui s'en dégage a permis le calcul numérique de ces points lorsque $X$ est une courbe modulaire (ou une courbe de Shimura), contexte dans lequel les travaux récents $[\mathbf{Y Z Z}]$ de X. Yuan, S. Zhang et W. Zhang les relient aux valeurs spéciales de certaines fonctions $L$ attachées à des formes modulaires.
\end{abstract}

2000 Mathematics Subject Classification. - 11F67; 11G05; 11G40; 11G18; 14C15.

Key words and phrases. - Pro-unipotent fundamental group, algebraic cycles, mixed Hodge structures, rational points, L-functions.

The exchanges which led to this work were initiated in a series of visits by the first (H.D) and third (I.S) author to the CRM in Barcelona on the occasion of its 2009-10 arithmetic special year which was organised in part by the second author (V.R). All three authors thank the CRM for its hospitality which greatly facilitated their collaboration. H.D. acknowledges the support of NSERC (Natural Sciences and Engineering Research Council of Canada). V.R. received financial support from DGICYT Grant MTM2009-13060-C02-01. I.S. also thanks the Spanish Ministerio de Educación for a Salvador Madariaga grant which funded his stay in the Institut de Recerca Matematica during the arithmetic special year. 


\section{Introduction}

Let $(Y, o)$ be a pointed algebraic variety over the complex numbers. Its pro-unipotent fundamental group $\pi_{1}(Y ; o)$ is a rich source of links between the topology, geometry and arithmetic of the variety: see for example the works of Bloch [Bl77], Chen [Chen], Deligne [De], Drinfeld [Dr], Hain [Hain3] and Kim [Ki] and the references therein.

Among these developments, the contributions of Carlson, Clemens and Morgan [CCM], Harris $[\mathbf{H a r}]$ and Pulte $[\mathbf{P u}]$ in the eighties exhibited examples where the values of Chen's iterated integrals -which provide an explicit description of the underlying mixed Hodge structure of the de Rham realization of $\pi_{1}(Y ; o)$ - coincide with the Abel-Jacobi images of a suitable nullhomologous cycle on $Y$ or on some other variety that one may naturally construct out of $Y$. In geometric terms, these results can be phrased as the equality of two points in an intermediate jacobian of the variety: one, constructed as the class of the periods afforded by certain iterated integrals, and the other obtained as the image of a suitable cycle under the Abel-Jacobi map.

Let $F \subset \mathbb{C}$ be a field embedded in the field of complex numbers, $X$ a smooth projective curve over $F$ and $Y=X \backslash\{\infty\}$ the complement of a single rational point $\infty \in X(F)$. Choose a base point $o \in Y(F)$. In this article we focus on the construction of a supply of complex points

$$
\kappa_{\mathrm{dR}}(\xi) \in \operatorname{Jac}(X)(\mathbb{C})
$$

on the jacobian of $X$, indexed by the set of Hodge classes

$$
\operatorname{Hdg}\left(H^{1}(X) \otimes H^{1}(X)\right):=\left\{\xi: \mathbb{Z}(-1) \rightarrow H^{1}(X) \otimes H^{1}(X)\right\}
$$

in the Hodge structure underlying $H^{1}(X) \otimes H^{1}(X)$. This construction has also been extensively studied by Pulte $[\mathbf{P u}]$ and Kaenders $[\mathbf{K a}]$ and we review it in $\S 1$. These points arise from the pro-unipotent fundamental group $\pi_{1}(Y(\mathbb{C}) ; o)$ and therefore depend on the choice of $o$, and their construction exploits the canonical isomorphism

$$
\operatorname{Ext}_{\mathrm{MHS}}^{1}\left(\mathbb{Z}(-1), H_{B}^{1}(X)\right) \simeq \frac{\Omega^{1}(X)^{\vee}}{H_{1}(X(\mathbb{C}), \mathbb{Z})} \simeq \operatorname{Jac}(X)(\mathbb{C})
$$

between the module of extensions of $\mathbb{Z}(-1)$ by $H_{\mathrm{B}}^{1}(X)$ in the category of mixed Hodge structures, and the set of complex points of $\operatorname{Jac}(X)$, as described e.g. in $[\mathbf{C a}]$.

Assume now that $\xi=\xi_{Z}$ is the Hodge class associated by the cycle class map with an algebraic cycle $Z \in \operatorname{Pic}(X \times X)$, as detailed in (44). Section 2 associates to each divisor $Z$ in $X \times X$ an algebraic correspondence $\Pi_{Z}: X^{3} \cdots \cdots, X$ between $X^{3}$ and $X$ and our first main theorem is:

Theorem 1. - For every divisor class $Z \in \operatorname{Pic}\left(X^{2}\right)$,

$$
\kappa_{\mathrm{dR}}\left(\xi_{Z}\right)=\Pi_{Z}\left(\Delta_{\mathrm{GKS}}\right) \in \operatorname{Jac}(X)(\mathbb{C})
$$

where

$-\Pi_{Z}: \mathrm{CH}^{2}\left(X^{3}\right)_{0} \longrightarrow \mathrm{CH}^{1}(X)_{0}=\operatorname{Jac}(X)$ is the transformation induced by $\Pi_{Z}$ by functoriality, and

- $\Delta_{\mathrm{GKS}} \in \mathrm{CH}^{2}\left(X^{3}\right)_{0}$ is the null-homologous cycle of codimension 2 in $X^{3}$ with base-point o studied by Gross, Kudla and Schoen [GrKu], [GrSc], cf. (47). 
This theorem spells out a relationship between diagonal cycles and the pro-unipotent fundamental group which is hinted at in the penultimate sentence of $\S 5.1$ of [ $\mathbf{Z h 2}$; the reader is encouraged to consult $\S 5$ of loc.cit. for further discussion of Gross-Kudla-Schoen cycles and their arithmetic applications. Since the right-hand side of (3) is purely algebraic, we derive the following corollary:

Corollary 2. - If $Z$ belongs to $\operatorname{Pic}(X \times X)(F)$ then $\kappa_{\mathrm{dR}}\left(\xi_{Z}\right)$ is an F-rational point of $\operatorname{Jac}(X)$.

The proof of Theorem 1 is complex analytic: it proceeds by relating-in $\S 1$ and $\S 2$, respectivelythe left and right-hand side of equality (3) to Chen's iterated integrals. The resulting explicit formula (Theorem 2.5) was one of the main motivations for this note and underlies the numerical calculations of Chow-Heegner points that are carried out in [DDLR] when $X$ is a modular curve. The authors also hope that Theorem 1 could form the basis for an eventual approach to the "Stark-Heegner points" of [DP] via iterated integrals.

Beyond the general expectation alluded to above that the pro-unipotent fundamental group $\pi_{1}(Y ; o)$ of a variety should encode the Abel-Jacobi images of non-trivial null-homologous algebraic cycles, it is also natural to expect, on the grounds of the conjectures of Bloch-Kato [BK] linking algebraic cycles to $L$-functions, that a good deal of the non-abelian arithmetic of $\pi_{1}(Y ; o)$ should be accounted for by the leading terms of the $L$-function of the motives appearing as Jordan-Hölder constituents of $\pi_{1}(Y ; o)$. There are however few examples in the literature in which this principle is illustrated ${ }^{(1)}$. A celebrated instance is afforded by the curve $Y=\mathbb{P}_{1}-\{0,1, \infty\}$, whose pro-unipotent fundamental group is an iterated extension of Tate motives. It is shown in [De] that the extension classes that arise in it encode special values of the Riemann zeta function at certain (non-critical) points. Theorem 3.7 in $\S 3$ describes analogous phenomena when $Y$ is a punctured modular curve attached to a congruence subgroup of $\mathrm{SL}_{2}(\mathbb{Z})$, or a Shimura curve, exploiting [YZZ].

Let us close this introduction by relating Theorem 1 to existing results in the literature. In our notations and the normalizations adopted in definition $(43)$ of the points $\kappa_{\mathrm{dR}}(\xi)-$ which are tailored so that there is no dependence on the choice of the point $\infty-$, the main theorem of Kaenders $[\mathbf{K a}]$ says that when $Z=X_{12} \in \operatorname{Pic}(X \times X)$ is the class of the curve $X$ diagonally embedded in $X \times X$, we have

$$
\kappa_{\mathrm{dR}}\left(\xi_{X_{12}}\right)=(2 g-2) o-K \in \operatorname{Jac}(X),
$$

where $g$ stands for the genus of $X$ and $K$ is any canonical divisor on the curve. Note that, since $K$ is an $F$-rational divisor, the conclusion of Corollary 2 follows directly from (4) for this particular choice of $Z$.

Kaenders proves (4) by showing that a suitable translate of $\kappa_{\mathrm{dR}}\left(\xi_{X_{12}}\right)$ is equal to twice Riemann's constant; his result then follows by an immediate application of the Riemann-Roch theorem. It can also be recovered directly from Theorem 1: see Corollary 2.8.

It is also interesting to compare Theorem 1 with the work of B. Harris [Har], as recast by Pulte in $[\mathbf{P u}]$. For any $n \in \mathbb{Z}$, let $[n] \in \operatorname{End}(\operatorname{Jac}(X))$ denote the endomorphism given by

1. while the connection between algebraic cycles and special values of $L$-functions as predicted by [BK] has already borne some fruit: cf. e.g. [GrZa], [Ne], [Zh], [YZZ]. 
multiplication-by- $n$. Define

$$
X_{o}:=\{[P-o]: P \in X\}, \quad X_{o}^{-}:=[-1]_{*} X_{o}=\{[o-P]: P \in X\} \subset \operatorname{Pic}^{0}(X)
$$

and let $C_{o}:=X_{o}-X_{o}^{-} \in \mathrm{CH}^{g-1}(\operatorname{Jac}(X))_{0}$ be Ceresa's cycle, a null-homologous cycle of codimension $g-1$ on the jacobian of $X$.

Every choice of Hodge class $\xi \in \operatorname{Hdg}\left(H_{B}^{1}(X)^{\otimes 2}\right)$ singles out a natural map ${ }^{(2)}$

$$
\pi_{\xi}: \mathrm{CH}^{g-1}(\operatorname{Jac}(X))_{0}(\mathbb{C}) \longrightarrow \operatorname{Jac}(X)(\mathbb{C})
$$

and Theorem 4.9 of $[\mathbf{P u}]$ asserts that

$$
\kappa_{\mathrm{dR}}(\xi)=2 \pi_{\xi}\left(C_{o}\right) \in \operatorname{Jac}(X)(\mathbb{C}) .
$$

Notice that Corollary 2 does not follow automatically from (6), as it is not a priori obvious that the map $\pi_{\xi}$ is Galois equivariant.

Our Theorem 1 is in the same spirit of Harris-Pulte's, in that both relate the points $\kappa_{\mathrm{dR}}(\xi)$ arising from $\pi_{1}(Y(\mathbb{C}) ; o$ ) to algebraic cycles on some algebraic variety intimately related to $X$, namely $X^{3}$ and $\operatorname{Jac}(X)$, respectively. These two varieties and their relevant Chow groups are related through their intermediate jacobians by the commutative diagram

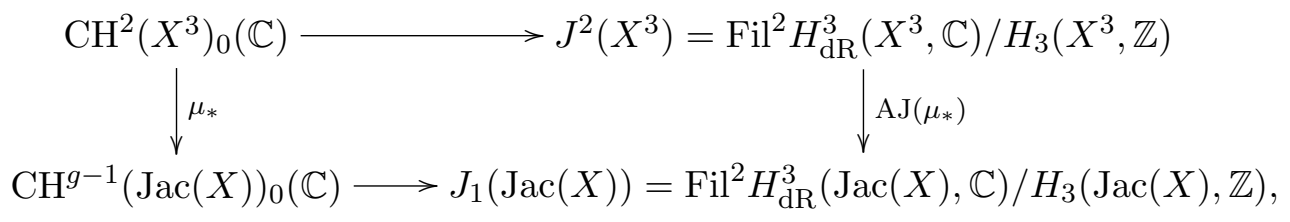

where the horizontal arrows are the Abel-Jacobi maps $\mathrm{AJ}_{X^{3}}$ on $X^{3}$ and $\mathrm{AJ}_{\mathrm{Jac}(X)}$ on $\operatorname{Jac}(X)$, respectively, the left-most vertical arrow is the transformation induced by functoriality by the morphism $\mu: X^{3} \rightarrow \operatorname{Jac}(X)$ given by the rule $\left(x_{1}, x_{2}, x_{3}\right) \mapsto x_{1}+x_{2}+x_{3}-3 o$, and the vertical arrow $\mathrm{AJ}(\mu)$ is the natural surjection given by the Künneth decomposition of $H^{3}\left(X^{3}\right)$ and the classical isomorphism $H^{3}(\operatorname{Jac}(X))=\Lambda^{3} H^{1}(X)$.

It readily follows from the definitions that

$$
\mu_{*}\left(\Delta_{\mathrm{GKS}}\right)=[3]_{*} X_{o}-3[2]_{*} X_{o}+3 X_{o} \in \mathrm{CH}^{g-1}(\operatorname{Jac}(X))_{0}
$$

and Colombo and van Geemen proved in [CG, Proposition 2.9] that

$$
\operatorname{AJ}_{\mathrm{Jac}(X)}\left(\mu_{*}\left(\Delta_{\mathrm{GKS}}\right)\right)=3 \mathrm{AJ}_{\mathrm{Jac}(X)}\left(C_{o}\right) .
$$

This result lies far from proving Theorem 1 via (6), as the maps $\mu_{*}$ and $\mathrm{AJ}\left(\mu_{*}\right)$ have non-trivial kernel. In fact, the combination of Theorem 1 with Pulte's (6) yields the equality

$$
\Pi_{Z}\left(\Delta_{\mathrm{GKS}}\right)=2 \pi_{\xi_{Z}}\left(C_{o}\right) \in \operatorname{Jac}(X)(\mathbb{C}) \quad \text { for all } Z \in \operatorname{Pic}(X \times X),
$$

which is yet another set of non-trivial relationships between Gross-Kudla-Schoen's cycle $\Delta_{\mathrm{GKS}} \in \mathrm{CH}^{2}\left(X^{3}\right)_{0}$ and Ceresa's $C_{o} \in \mathrm{CH}^{g-1}(\operatorname{Jac}(X))_{0}$.

2. In the notation of $\S 1.2, \pi_{\xi}$ is the composition of the Abel-Jacobi map $\mathrm{CH}^{g-1}(\operatorname{Jac}(X))_{0}(\mathbb{C}) \stackrel{\text { AJ }}{\rightarrow} J_{1}(\operatorname{Jac}(X))$, the monomorphism $J_{1}(\operatorname{Jac}(X)) \hookrightarrow \operatorname{Ext}_{\mathrm{MHS}}^{1}\left(H_{B}^{1}(X)^{\otimes 2}, H_{B}^{1}(X)\right)$ considered by Pulte in $[\mathbf{P u}]$, the projection $\operatorname{Ext}_{\mathrm{MHS}}^{1}\left(H_{B}^{1}(X)^{\otimes 2}, H_{B}^{1}(X)\right) \rightarrow \operatorname{Ext}_{\mathrm{MHS}}^{1}\left(\mathbb{Z}(-1), H_{B}^{1}(X)\right)$ given by $\xi$ as in (36) and the isomorphism (2). 


\section{Fundamental groups and mixed motives}

Let $F$ be a number field equipped with an embedding $F \hookrightarrow \mathbb{C}$ into the field of complex numbers, and write $\bar{F}$ for the Galois closure of $F$ in $\mathbb{C}$; as before, recall that $G_{F}:=\operatorname{Gal}(\bar{F} / F)$. Let $X$ be a smooth, complete algebraic curve of genus $g \geq 2$ over $F$, and let $Y=X \backslash S$ for some finite, $G_{F}$-stable set $S$ of points on $X(\bar{F})$. Fix a base point $o \in Y(F)$; the integral group ring $\mathbb{Z}[\Gamma]$ of the fundamental group $\Gamma:=\pi_{1}(Y(\mathbb{C}) ; o)$ of the Riemann surface underlying

$$
Y_{\mathbb{C}}=Y \times_{\operatorname{Spec}(F)} \operatorname{Spec}(\mathbb{C})
$$

is equipped with a decreasing filtration

$$
\mathbb{Z}[\Gamma] \supset I \supset I^{2} \supset \cdots \supset I^{j} \supset \cdots
$$

by powers of the augmentation ideal.

The first few successive quotients in this filtration are given by

$$
\begin{aligned}
\mathbb{Z}[\Gamma] / I=\mathbb{Z} ; & \sum m_{\sigma} \sigma \mapsto \sum m_{\sigma} ; \\
I / I^{2}=\Gamma_{\mathrm{ab}}:=\Gamma /[\Gamma, \Gamma] ; & \sum m_{\sigma} \sigma \mapsto \prod \sigma^{m_{\sigma}} ; \\
I^{2} / I^{3}=\left(\Gamma_{\mathrm{ab}} \otimes \Gamma_{\mathrm{ab}}\right) / \mathcal{K}, &
\end{aligned}
$$

where in the last identification, $\mathcal{K}$ is the kernel of the natural surjective homomorphism

$$
j: \Gamma_{\mathrm{ab}} \otimes \Gamma_{\mathrm{ab}} \longrightarrow I^{2} / I^{3}, \quad \gamma_{1} \otimes \gamma_{2} \mapsto\left(\gamma_{1}-1\right)\left(\gamma_{2}-1\right) .
$$

Let $M^{\vee}:=\operatorname{hom}(M, \mathbb{Z})$ be the $\mathbb{Z}$-linear dual of a $\mathbb{Z}$-module $M$. Formula (11) implies that

$$
\left(I / I^{2}\right)^{\vee}=\operatorname{hom}(\Gamma, \mathbb{Z})=H_{\mathrm{B}}^{1}(Y),
$$

where $H_{\mathrm{B}}^{1}(Y)=H^{1}(Y(\mathbb{C}), \mathbb{Z})$ refers to the singular Betti cohomology of the smooth real manifold underlying $Y(\mathbb{C})$. Furthermore, it can be shown that the map

$$
j^{\vee}:\left(I^{2} / I^{3}\right)^{\vee} \hookrightarrow H_{\mathrm{B}}^{1}(Y)^{\otimes 2}
$$

identifies $\left(I^{2} / I^{3}\right)^{\vee}$ with the kernel of the map

$$
H_{\mathrm{B}}^{1}(Y)^{\otimes 2} \longrightarrow H_{\mathrm{B}}^{2}(Y)
$$

given by the cup product. (Cf. [Hain3, Cor. 8.2] for more details.) Letting $H_{\mathrm{B}}^{1}(Y) \underset{\cup=0}{\otimes 2}$ denote this kernel, the map $j^{\vee}$ induces an identification

$$
\left(I^{2} / I^{3}\right)^{\vee}=H_{\mathrm{B}}^{1}(Y) \stackrel{\otimes}{\cup=0} \text {. }
$$

By dualising the exact sequence

$$
0 \longrightarrow I^{2} / I^{3} \longrightarrow I / I^{3} \longrightarrow I / I^{2} \longrightarrow 0,
$$

invoking (14) and (15), and setting

$$
M_{\mathrm{B}}:=\left(I / I^{3}\right)^{\vee},
$$

we obtain the fundamental exact sequence of $\mathbb{Z}$-modules

$$
0 \longrightarrow H_{\mathrm{B}}^{1}(Y) \longrightarrow M_{\mathrm{B}} \longrightarrow H_{\mathrm{B}}^{1}(Y) \cup \cup^{\otimes 2} \longrightarrow 0 \text {. }
$$

Note that $H_{\mathrm{B}}^{1}(Y) \cup 2=H_{\mathrm{B}}^{1}(Y)^{\otimes 2}$ as soon as $S \neq \emptyset$, because in this case $H_{\mathrm{B}}^{2}(Y)=\{0\}$. The abelian groups that appear on the left and right of (16) are not just "unadorned" $\mathbb{Z}$-modules, but the Betti realisations of certain motives over $F$. In the terminology of [De], the exact 
sequence (16) suggests that $M_{B}$ ought to be interpreted as the Betti realization of a putative mixed motive $M^{?}$ (in the sense of [De], say) which would arise as an extension in that category of the motive $H^{1}(Y) \cup 2$ (which is pure of weight 2 when $Y=X$ ) by the motive $H^{1}(Y$ ) (which is pure of weight 1 when $Y=X)$. Implicit in the existence of $M^{\text {? }}$, which we do not address in this paper, are the following concrete consequences:

1. The existence of a decreasing, separated and exhaustive Hodge filtration on

$$
M_{\mathrm{dR}, \mathbb{C}}:=M_{\mathrm{B}} \otimes \mathbb{C}
$$

for which the complexification

$$
0 \longrightarrow H_{\mathrm{dR}}^{1}(Y / \mathbb{C}) \stackrel{i}{\longrightarrow} M_{\mathrm{dR}, \mathbb{C}} \stackrel{\mathrm{p}}{\longrightarrow} H_{\mathrm{dR}}^{1}(Y / \mathbb{C})_{\cup=0}^{\otimes 2} \longrightarrow 0
$$

of (16) becomes an exact sequence of filtered complex vector spaces.

2. The existence, for each rational prime $p$, of the $p$-adic étale realisation $M_{\text {et, } p}$, fitting into an exact sequence of continuous $\mathbb{Q}_{p}\left[G_{F}\right]$-modules

$$
0 \longrightarrow H_{\text {et }}^{1}\left(X_{\bar{F}}, \mathbb{Q}_{p}\right) \longrightarrow M_{\text {et }, p} \longrightarrow H_{\text {et }}^{1}\left(X_{\bar{F}}, \mathbb{Q}_{p}\right) \stackrel{\otimes 2}{\otimes=0} \longrightarrow 0
$$

which is the $p$-adic étale counterpart of (16).

There are other aspects as well (notably, the cristalline realisations attached to the fundamental groups of the reduction of $Y$ modulo $p$, equipped with its semi-linear frobenius endomorphism at $p$ and a comparison isomorphism with the de Rham cohomology over $p$-adic fields) which shall not be touched upon at all here. In this note we focus almost exclusively on (17).

Let us briefly recall the well-known mixed Hodge structures underlying the Betti cohomology group $H_{\mathrm{B}}^{1}(Y)$ and its tensor square. Its weight filtration arises from the exact sequence

$$
0 \longrightarrow H_{\mathrm{B}}^{1}(X) \longrightarrow H_{\mathrm{B}}^{1}(Y) \longrightarrow \oplus_{P \in S} \mathbb{Z}(-1) \longrightarrow \mathbb{Z}(-1) \longrightarrow 0
$$

and is given by

$$
W_{j} H_{\mathrm{B}}^{1}(X)= \begin{cases}0 & \text { if } j \leq 0 \\ H_{\mathrm{B}}^{1}(X) & \text { if } j=1 \\ H_{\mathrm{B}}^{1}(Y) & \text { if } j \geq 2\end{cases}
$$

The de Rham cohomology $H_{\mathrm{dR}}^{1}(Y(\mathbb{C}))$ is the cohomology of the de Rham complex of smooth $\mathbb{C}$-valued differential forms on $Y(\mathbb{C})$ with at worst logarithmic poles at the points in $S$. There is a canonical identification $H_{\mathrm{B}}^{1}(Y) \otimes \mathbb{C}=H_{\mathrm{dR}}^{1}(Y(\mathbb{C}))$ arising from integration of smooth differential forms against smooth chains, and its Hodge filtration is given by

$$
\mathrm{Fil}^{j} H_{\mathrm{dR}}^{1}(Y(\mathbb{C}))= \begin{cases}H_{\mathrm{dR}}^{1}(Y(\mathbb{C})) & \text { if } j \leq 0, \\ \Omega_{\log }^{1}(Y(\mathbb{C})) & \text { if } j=1, \\ 0 & \text { if } j \geq 2,\end{cases}
$$

where

$$
\Omega_{\log }^{1}(Y(\mathbb{C}))=\left\{\omega \in \Omega^{1}(Y(\mathbb{C})) \quad \text { with } \quad \operatorname{ord}_{P}(\omega) \geq-1, \quad \forall P \in S\right\}
$$

and $\operatorname{ord}_{\mathrm{P}}(\omega)$ denotes the valuation of the differential $\omega$ at the point $P \in X(\mathbb{C})$.

Because the Riemann surface $Y(\mathbb{C})$ arises from an algebraic curve over $F$, we can identify $H_{\mathrm{dR}}^{1}(Y(\mathbb{C}))$ with $H_{\mathrm{dR}}^{1}(Y / F) \otimes \mathbb{C}$, where

$$
H_{\mathrm{dR}}^{1}(Y / F):=\mathbb{H}^{1}\left(0 \rightarrow \mathcal{O}_{Y} \rightarrow \Omega^{1}(Y) \rightarrow 0\right)
$$


is the algebraic de Rham cohomology of $Y / F$, defined as the hypercohomology of the de Rham complex of sheaves of regular differential forms on $Y$. The Hodge filtration on $H_{\mathrm{dR}}^{1}(Y / F)$ may also be described concretely by

$$
\mathrm{Fil}^{j} H_{\mathrm{dR}}^{1}(Y / F)= \begin{cases}H_{\mathrm{dR}}^{1}(Y / F) \simeq \Omega^{I I}(Y) / d F(Y) & \text { if } j \leq 0, \\ \Omega_{\log }^{1}(Y / F) & \text { if } j=1, \\ 0 & \text { if } j \geq 2,\end{cases}
$$

where

1. $\Omega^{I I}(Y)$ is the space of rational differential forms on $Y$ over $F$ with vanishing residues at all points of $Y$; these are also called differentials of the second kind on $Y$;

2. $F(Y)$ is the field of rational functions on $Y$ over $F$;

3. $\Omega_{\log }^{1}(Y / F)$ is the subspace of $\Omega^{1}(Y / F)$ consisting of differentials $\omega$ satisfying $\operatorname{ord}_{P}(\omega) \geq$ -1 for all $P \in S$.

The tensor product $H_{\mathrm{B}}^{1}(Y)^{\otimes 2}$ is again a mixed Hodge structure, and the Hodge filtration on $H_{\mathrm{dR}}^{1}(Y / F)^{\otimes 2}$ is given by

$$
\mathrm{Fil}^{j} H_{\mathrm{dR}}^{1}(Y / F)^{\otimes 2}= \begin{cases}H_{\mathrm{dR}}^{1}(Y / F)^{\otimes 2} & \text { if } j \leq 0 ; \\ \Omega_{\log }^{1}(Y / F) \otimes H_{\mathrm{dR}}^{1}(Y / F)+H_{\mathrm{dR}}^{1}(Y / F) \otimes \Omega_{\log }^{1}(Y / F) & \text { if } j=1 ; \\ \Omega_{\log }^{1}(Y / F)^{\otimes 2} & \text { if } j=2 ; \\ 0 & \text { if } j \geq 3 .\end{cases}
$$

1.1. Iterated integrals and the Hodge filtration on $M_{\mathrm{dR}, \mathbb{C}}$ - - The extension $M_{B}$ of $H_{\mathrm{B}}^{1}(Y)^{\otimes 2}$ by $H_{\mathrm{B}}^{1}(Y)$ arising in (16) is equipped with a natural mixed Hodge structure. The definition of the Hodge filtration on $M_{\mathrm{dR}, \mathbb{C}}$ was given by R. Hain in [Hain2], and rests on the explicit description of $M_{\mathrm{dR}, \mathbb{C}}$ in terms of K.-T. Chen's iterated integrals of smooth differential forms on path spaces. We now briefly recall this description, referring the reader to [Chen], [Hain3] and the references therein for further details.

The path space on $Y$ based at $o$, denoted $\mathbb{P}(Y ; o)$, is the set of piecewise smooth paths

$$
\gamma:[0,1] \longrightarrow Y(\mathbb{C}), \quad \text { with } \gamma(0)=o .
$$

The universal covering space of $Y(\mathbb{C})$, denoted $\tilde{Y}$, is the space of homotopy classes in $\mathbb{P}(Y ; o)$. It is conformally equivalent to the open unit disc in $\mathbb{C}$. The group $\Gamma=\pi_{1}(Y(\mathbb{C}) ; o)$ acts on it transitively and without fixed points, and the map $\gamma \mapsto \gamma(1)$ identifies the quotient $\tilde{Y} / \Gamma$ with $Y(\mathbb{C})$. If $\eta$ is a smooth closed one-form (resp. a meromorphic one-form of the second kind) on $Y(\mathbb{C})$, then it admits a smooth (resp. meromorphic) primitive $F_{\eta}$ on $\tilde{Y}$, defined by the rule

$$
F_{\eta}(\gamma):=\int_{0}^{1} \gamma^{*} \eta
$$

The basic iterated integral attached to smooth differential forms $\omega_{1}, \ldots, \omega_{n}$ along a path $\gamma \in \mathbb{P}(Y ; o)$ is defined to be

$$
\int_{\gamma} \omega_{1} \cdot \omega_{2} \cdots \omega_{n}:=\int_{0 \leq t_{n} \leq t_{n-1} \leq \cdots \leq t_{1} \leq 1} \gamma^{*}\left(\omega_{1}\right)\left(t_{1}\right) \cdots \gamma^{*}\left(\omega_{n}\right)\left(t_{n}\right) .
$$


The integer $n$ is called the length of this basic iterated integral. In particular, when $n=2$, the basic iterated integral attached to $\eta_{1}$ and $\eta_{2}$ is given by the formula

$$
\int_{\gamma} \eta_{1} \cdot \eta_{2}:=\int_{\gamma} \eta_{1} F_{\eta_{2}}=\int_{0}^{1} \gamma^{*}\left(\eta_{1} F_{\eta_{2}}\right)
$$

It can also be expressed in terms of $\eta_{1} \wedge \eta_{2}$, viewed as a two-form on $X \times X$, as the expression

$$
\int_{\gamma} \eta_{1} \cdot \eta_{2}:=\int_{T_{\gamma}} \eta_{1} \wedge \eta_{2}, \quad \text { where } T_{\gamma}=\{(\gamma(r), \gamma(s)), \quad 0 \leq s \leq r \leq 1\} \subset X \times X \text {. }
$$

An iterated integral is any linear combination of basic iterated integrals, of possibly differing lengths, viewed as a function on $\mathbb{P}(Y ; o)$. The length of an iterated integral is then defined to be the maximal length arising in its expression as a linear combination of basic iterated integrals.

An iterated integral is said to be homotopy invariant if its value on a path $\gamma$ depends only on the homotopy class of $\gamma$. The space $\operatorname{II}(Y)$ of homotopy invariant iterated integrals will be viewed as a subspace of the space of $\mathbb{C}$-valued functions on $\Gamma$, in the obvious way. By extending $J \in \mathrm{II}(Y)$ to the group ring $\mathbb{C}[\Gamma]$ by $\mathbb{C}$-linearity, we will exploit the resulting natural inclusion

$$
\mathrm{II}(Y) \subset \mathbb{C}[\Gamma]^{\vee}
$$

to realise $\mathrm{II}(Y)$ as a space of complex functionals on $\mathbb{C}[\Gamma]$. For each $n$, let $\mathrm{II} \leq n(Y)$ denote the subspace of homotopy invariant iterated integrals of length $\leq n$. This space plays an important role in our description of $M_{\mathrm{dR}, \mathbb{C}}$, because of the following facts:

1. Any element $J \in \mathrm{II}^{\leq n}(Y) \subset \mathbb{C}[\Gamma]^{\vee}$ vanishes on $I^{n+1}$, and hence gives rise to a welldefined element of $\left(I / I^{n+1}\right)^{\vee} \otimes_{\mathbb{Z}} \mathbb{C}$.

2. The natural map

$$
\mathrm{II} \leq n \longrightarrow\left(I / I^{n+1}\right)^{\vee} \otimes_{\mathbb{Z}} \mathbb{C}
$$

is an isomorphism. In particular,

$$
M_{\mathrm{dR}, \mathbb{C}}=\mathrm{II}^{\leq 2}(Y) .
$$

The following lemma is helpful in describing $\mathrm{II} \leq 2(Y)$ concretely.

Lemma 1.1. - Let $\eta_{1}$ and $\eta_{2}$ be smooth closed one-forms on $Y(\mathbb{C})$ whose classes in de Rham cohomology are orthogonal with respect to the Poincaré pairing, and let $\alpha$ be a smooth one-form on $Y(\mathbb{C})$ satisfying the differential equation

$$
d \alpha=\eta_{1} \wedge \eta_{2}
$$

1. The iterated integral

$$
J_{\eta_{1} \otimes \eta_{2}, \alpha}(\gamma):=\int_{\gamma}\left(\eta_{1} \cdot \eta_{2}-\alpha\right)
$$

is homotopy invariant.

2. The image of $J_{\eta_{1} \otimes \eta_{2}, \alpha} \in M_{\mathrm{dR}, \mathbb{C}}$ under the projection $\mathrm{p}$ of $(17)$ is the element $\left[\eta_{1}\right] \otimes\left[\eta_{2}\right]$.

3. The space $\mathrm{II} \leq 2(Y)$ is spanned by iterated integrals of the form $J_{\eta_{1} \otimes \eta_{2}, \alpha}$. 
Proof. - The first claim follows directly from the fact that the differential one-form on $\tilde{Y}$ appearing in the expression

$$
J_{\eta_{1} \otimes \eta_{2}, \alpha}(\gamma)=\int_{\gamma} \eta_{1} F_{\eta_{2}}-\alpha
$$

is closed. The second statement follows from a direct calculation showing that

$$
J_{\eta_{1} \otimes \eta_{2}, \alpha}\left(\left(\gamma_{1}-1\right)\left(\gamma_{2}-1\right)\right)=\int_{\gamma_{1}} \eta_{1} \int_{\gamma_{2}} \eta_{2} .
$$

Finally, given any $J \in M_{\mathrm{dR}, \mathbb{C}}$, after writing $\mathrm{p}(J)=\sum \omega_{i} \otimes \eta_{i}$, it can be checked that

$$
J=\left(\sum J_{\omega_{i} \otimes \eta_{i}, \alpha_{i}}\right)-J_{0,0, \alpha}
$$

for suitable smooth differentials $\alpha_{i}$ on $Y(\mathbb{C})$ and $\alpha \in H_{\mathrm{dR}}^{1}(Y(\mathbb{C}))$.

In the special case where $Y(\mathbb{C})=X(\mathbb{C})-\{\infty\}$ with $X$ projective, the smooth differential $\alpha$ on $Y(\mathbb{C})$ can be expressed in terms of the Green's function $g_{\eta_{1} \wedge \eta_{2}, \infty}$ attached to the smooth $(1,1)$-form $\eta_{1} \wedge \eta_{2}$ and the point $\infty$. Recall (cf. [La], §II.1 for example) that $g_{\eta_{1} \wedge \eta_{2}, \infty}$ is a smooth $\mathbb{C}$-valued function on $Y(\mathbb{C})$ satisfying the following two conditions:

1. Letting $\partial$ and $\bar{\partial}: \mathcal{O}_{\text {smooth }}(Y) \longrightarrow \Omega_{\text {smooth }}^{1}(Y)$ be the usual differential operators defined by

$$
\partial f=\frac{\partial f}{\partial z} d z=\frac{1}{2}\left(\frac{\partial f}{\partial x}-i \frac{\partial f}{\partial y}\right)(d x+i d y), \quad \bar{\partial} f=\frac{\partial f}{\partial \bar{z}} d \bar{z}=\frac{1}{2}\left(\frac{\partial f}{\partial x}+i \frac{\partial f}{\partial y}\right)(d x-i d y)
$$

the function $g_{\eta_{1} \wedge \eta_{2}, \infty}$ is a solution of the differential equation

$$
\frac{-1}{2 \pi i} \partial \bar{\partial} g_{\eta_{1} \wedge \eta_{2}, \infty}=\eta_{1} \wedge \eta_{2}
$$

2. The function $g_{\eta_{1} \wedge \eta_{2}}$ has at worst logarithmic growth at $\infty$. More precisely, letting

$$
m_{\eta_{1} \wedge \eta_{2}}:=\int_{X(\mathbb{C})} \eta_{1} \wedge \eta_{2}
$$

the function $g_{\eta_{1} \wedge \eta_{2}, \infty}$ can be written as

$$
g_{\eta_{1} \wedge \eta_{2}, \infty}(x)=-m_{\eta_{1} \wedge \eta_{2}} \log \left|f_{\infty}(x)\right|^{2}+h(x),
$$

where $f_{\infty}$ is a rational function on $X$ with a simple zero at $\infty$ and $h(x)$ is smooth in a neighbourhood of $\infty$.

The differential

$$
\alpha_{\infty}:=\frac{1}{2 \pi i} \partial g_{\eta_{1} \wedge \eta_{2}, \infty}
$$

is a smooth one-form of type $(1,0)$ on $Y(\mathbb{C})$ satisfying

$$
d \alpha_{\infty}=(\partial+\bar{\partial}) \alpha_{\infty}=\bar{\partial} \alpha_{\infty}=\eta_{1} \wedge \eta_{2}
$$

Its Laurent development around $\infty$ has a principal part of the form

$$
\alpha_{\infty}=\frac{-m_{\eta_{1} \wedge \eta_{2}}}{2 \pi i} \frac{d z}{z}
$$


This implies that, for any smooth region $R \subset X(\mathbb{C})$ whose boundary does not contain $\infty$,

$$
\int_{R} \eta_{1} \wedge \eta_{2}=\int_{\partial R} \alpha_{\infty}+m_{\eta_{1} \wedge \eta_{2}} \int_{R} \delta_{\infty}
$$

where $\delta_{\infty}$ is the Dirac delta current, satisfying

$$
\int_{R} \delta_{\infty}= \begin{cases}1 & \text { if } \infty \in R \\ 0 & \text { if } \infty \notin R\end{cases}
$$

In other words, we have the equality of currents:

$$
\eta_{1} \wedge \eta_{2}=d \alpha_{\infty}+m_{\eta_{1} \wedge \eta_{2}} \delta_{\infty}
$$

The following lemma concerning the differential $\alpha_{\infty}$ shall be used in the proofs of Section 1.2.

Lemma 1.2. - After setting $X=X_{1}=X_{2}$, viewing $\eta_{1} \wedge \eta_{2}$ as a $(1,1)$-form on $X_{1}$, and letting $\rho$ be a closed one-form on $X_{2}$, consider the three-form $\left(\eta_{1} \wedge \eta_{2}\right) \wedge \rho$ on $X_{1} \times X_{2}$. Let $W$ be any smooth three-chain on $X_{1} \times X_{2}$ which intersects $\{\infty\} \times X_{2}$ in a smooth one-chain, so that

$$
(\partial W)^{\prime}:=\partial W-\partial\left(W \cap\left(\{\infty\} \times X_{2}\right)\right)
$$

is the complement of a finite set in $\partial W$. Then the integral

$$
\int_{\partial W} \alpha_{\infty} \wedge \rho:=\int_{(\partial W)^{\prime}} \alpha_{\infty} \wedge \rho
$$

converges, and satisfies

$$
\int_{W}\left(\eta_{1} \wedge \eta_{2}\right) \wedge \rho=\int_{\partial W} \alpha_{\infty} \wedge \rho+m_{\eta_{1} \wedge \eta_{2}} \int_{W \cap\left(\{\infty\} \times X_{2}\right)} \rho .
$$

Proof. - Since $\rho$ is closed, equation (30) implies that

$$
\left(\eta_{1} \wedge \eta_{2}\right) \wedge \rho=d\left(\alpha_{\infty} \wedge \rho\right)+m_{\eta_{1} \wedge \eta_{2}} \cdot \delta_{\infty} \wedge \rho .
$$

Integrating over $W$ and applying Stokes' theorem, we obtain

$$
\int_{W}\left(\eta_{1} \wedge \eta_{2}\right) \wedge \rho=\int_{\partial W} \alpha_{\infty} \wedge \rho+m_{\eta_{1} \wedge \eta_{2}} \int_{W} \delta_{\infty} \wedge \rho,
$$

and the lemma follows from the fact that $\int_{W} \delta_{\infty} \wedge \rho=\int_{W \cap\left(\{\infty\} \times X_{2}\right)} \rho$.

Following Hain (cf. [Hain3, §13.1]), the Hodge filtration on $\left(I / I^{n+1}\right)^{\vee} \otimes_{\mathbb{Z}} \mathbb{C}$ is given in terms of $(26)$ by defining $\operatorname{Fil}^{j}\left(\left(I / I^{n+1}\right)^{\vee} \otimes_{\mathbb{Z}} \mathbb{C}\right)$ to be the set of $J \in \mathrm{II}^{\leq n}$ of the form

$$
J=\left(\int \omega_{1}^{(1)} \cdot \omega_{2}^{(1)} \cdots \omega_{n_{1}}^{(1)}\right)+\cdots+\left(\int \omega_{1}^{(t)} \cdot \omega_{2}^{(t)} \cdots \omega_{n_{t}}^{(t)}\right)
$$

in which each $n_{i}$-tuple $\left(\omega_{1}^{(i)}, \ldots, \omega_{n_{i}}^{(i)}\right)$ involves at least $j$ smooth differential forms of type $(1,0)$ on $Y(\mathbb{C})$ with logarithmic growth along $S$. In particular, for $n=2$ we have

(i) $\mathrm{Fil}^{j} M_{\mathrm{dR}, \mathbb{C}}=M_{\mathrm{dR}, \mathbb{C}}$ for all $j \leq 0$;

(ii) The space $\mathrm{Fil}^{1} M_{\mathrm{dR}, \mathbb{C}}$ is spanned by iterated integrals of the form $J_{\eta_{1}, \eta_{2}, \alpha}$ in which $\alpha$ and at least one of $\eta_{1}$ and $\eta_{2}$ lie in $\Omega_{\log }^{1}(Y(\mathbb{C}))$. 
(iii) The space $\mathrm{Fil}^{2} M_{\mathrm{dR}, \mathbb{C}}$ is spanned by iterated integrals of the form $J_{\omega_{1}, \omega_{2}, 0}$ in which $\omega_{1}$ and $\omega_{2}$ both belong to $\Omega_{\log }^{1}(Y(\mathbb{C}))$.

(iv) For all $j \geq 3$, Fil $^{j} M_{\mathrm{dR}, \mathbb{C}}=0$.

With this description of the Hodge filtration on $M_{\mathrm{dR}, \mathbb{C}}$, it is routine to verify that the exact sequence (17) is an exact sequence of filtered vector spaces. The $\mathbb{Z}$-module $M_{\mathrm{B}}$ is in fact a prototypical example of an integral mixed Hodge structure. (See [Hain3, Cor. 9.3] and [Hain1] for a discussion in a more general setting.) Let

$$
\kappa_{\mathrm{dR}} \in \operatorname{Ext}_{\mathrm{MHS}}^{1}\left(H_{\mathrm{B}}^{1}(Y) \stackrel{\otimes 2}{\cup=0}, H_{\mathrm{B}}^{1}(Y)\right)
$$

denote the class of the extension (16) in the category MHS of mixed Hodge structures. We will primarily be concerned with understanding this extension class and finding criteria for it to be non-trivial.

\section{An alternate description of the Hodge filtration}

The analytic description of the Hodge filtration on $M_{\mathrm{dR}, \mathbb{C}}$ which we have just given is wellsuited for the theoretical calculations of this note. However, the difficulties inherent in solving equation (28) seem to make it ill-suited for the practical numerical calculations of Abel-Jacobi maps that are carried out in [DDLR]. For the latter, it is convenient to exploit an algebraic description of $M_{\mathrm{dR}, \mathbb{C}}$ and its Hodge filtration involving meromorphic differentials of the second kind rather than smooth differentials.

Note that the definitions of $\mathrm{Fil}^{j} M_{\mathrm{dR}, \mathbb{C}}$ given above apply just as well to the algebraic setting, with the exception of $\mathrm{Fil}^{1} M_{\mathrm{dR}, \mathbb{C}}$ which is described in terms of smooth forms. We now proceed to give a description of this space involving meromorphic differentials on $Y$ of the second kind. Firstly, given $\omega \in \Omega_{\log }^{1}(Y(\mathbb{C}))$ and a smooth closed one-form $\eta$ on $Y(\mathbb{C})$, the two-form $\omega \wedge \eta+$ $\eta \wedge \omega$ vanishes identically on $Y$. In particular, the class of $\omega \otimes \eta+\eta \otimes \omega$ always belongs to $H^{1}(Y) \cup 2$, even when $Y$ is projective, and the elements of $M_{\mathrm{dR}, \mathbb{C}}$ which map to this class are those of the form

$$
\begin{array}{rc}
J_{\omega \otimes \eta+\eta \otimes \omega, \alpha}, & \text { with } d \alpha=0 ; \\
J_{\omega \otimes \eta+\eta \otimes \omega, \alpha}(\gamma):= & \int_{\gamma}(\omega \cdot \eta+\eta \cdot \omega)-\int_{\gamma} \alpha .
\end{array}
$$

A direct calculation reveals that

$$
J_{\omega \otimes \eta+\eta \otimes \omega, \alpha}(\gamma)=\left(\int_{\gamma} \omega\right)\left(\int_{\gamma} \eta\right)-\int_{\gamma} \alpha,
$$

so that the values of $J_{\omega \otimes \eta+\eta \otimes \omega, \alpha}$ are expressed in terms of products of periods of $\gamma$ relative to the de Rham cohomology classes of $\omega, \eta$ and $\alpha$. The function $J_{\omega \otimes \eta+\eta \otimes \omega, \alpha}$ can therefore be readily calculated in practice using meromorphic representatives of the classes of $\omega, \eta$, and $\alpha$. It is then clear from the description of the Hodge filtration on $M_{\mathrm{dR}, \mathbb{C}}$ given in (ii) above that

$$
J_{\omega \otimes \eta+\eta \otimes \omega, \alpha} \text { belongs to } \mathrm{Fil}^{1} M_{\mathrm{dR}, \mathbb{C}} \text { if and only if } \alpha \in \Omega_{\log }^{1}(Y / \mathbb{C}) .
$$

Secondly, let us represent a cohomology class $\xi \in H_{\mathrm{dR}}^{1}(Y / \mathbb{C})$ by a meromorphic differential form $\eta$ of the second kind on $Y(\mathbb{C})$ with at worst logarithmic poles along $S$, making use of the algebraic description for $H_{\mathrm{dR}}^{1}(Y / F)$ given in (23). The principal part of the meromorphic differential 1-form $\omega F_{\eta}$ at a point $x \in \tilde{Y}$, denoted $\underline{\mathrm{pp}}_{x}\left(\omega F_{\eta}\right)$, depends only on the image of $x$ 
in $Y(\mathbb{C})$. Indeed, let $\gamma x \in \tilde{Y}$, for $\gamma \in \Gamma$, be any other point in $\tilde{Y}$ lying above the same point of $Y(\mathbb{C})$. Comparing principal parts, we find:

$$
\underline{\mathrm{pp}}_{\gamma x}\left(\omega F_{\eta}\right)=\underline{\mathrm{pp}}_{x}\left(\gamma^{*}\left(\omega F_{\eta}\right)\right)=\underline{\mathrm{pp}}_{x}\left(\omega F_{\eta}+c \omega\right)
$$

for some constant $c$, and the claim follows because $\omega$ is regular. In particular, it makes sense to talk about the principal part $\underline{\mathrm{pp}}_{P}\left(\omega F_{\eta}\right)$ at a point $P \in Y(\mathbb{C})$, even though the one-form $\omega F_{\eta}$ is only defined on $\tilde{Y}$. A direct application of the Riemann-Roch theorem shows that there exists a meromorphic differential form $\alpha$ on $Y(\mathbb{C})$ with at worst logarithmic poles along $S$, satisfying

$$
\underline{\operatorname{pp}}_{P}(\alpha)=\underline{\operatorname{pp}}_{P}\left(\omega \cdot F_{\eta}\right), \quad \text { for all } P \in Y(\mathbb{C}) .
$$

The one-form $\omega \cdot F_{\eta}-\alpha$ is then holomorphic on $\tilde{Y}$. The differentials $\alpha$ satisfying (33) all differ by elements of $\Omega_{\log }^{1}(Y(\mathbb{C}))=\operatorname{Fil}^{1} H_{\mathrm{dR}}^{1}(Y / \mathbb{C})$, and we again have that

$$
J_{\omega \otimes \eta, \alpha} \text { belongs to } \mathrm{Fil}^{1} M_{\mathrm{dR}, \mathbb{C}} \text {. }
$$

It is then obvious from the definition of $\mathrm{Fil}^{1} M_{\mathrm{dR}, \mathbb{C}}$ given in (ii) above that this space can be recovered in terms of meromorphic differentials as the vector space generated by the iterated integrals appearing in (32) and (34).

Given a regular differential $\omega$ and a meromorphic differential $\eta$ of the second kind on $Y$, the task of calculating the meromorphic one-form $\alpha$ satisfying (33) can be carried out explicitly in the case of modular curves, and seems easier in practice than solving the equation (28) associated to a smooth representative of the class of $\eta$. See the forthcoming article [DDLR] for a more detailed discussion of these computational aspects.

1.2. Points arising from Hodge classes and the fundamental group. — The goal of this section is to recall a construction which goes back at least to $[\mathbf{K a}]$ and $[\mathbf{P u}]$ whereby the extension class $\kappa_{\mathrm{dR}}$ of (31) arising from the pro-unipotent fundamental group are used to construct rational points in the Jacobian of a (smooth, projective) curve ${ }^{(3)}$. We retain the same notations as in the previous section and assume that $Y=X \backslash\{\infty\}$ is the complement of a point $\infty \in X(F)$.

A Hodge class in a mixed Hodge structure $N$ of weight two is a morphism

$$
\xi: \mathbb{Z}(-1) \longrightarrow N
$$

of mixed Hodge structures, where $\mathbb{Z}(-1)$ denotes the weight 2 Hodge structure attached to the Tate motive $H^{2}\left(\mathbb{P}_{1}\right)$. The $\mathbb{Z}$-module of all such Hodge classes is denoted $\operatorname{Hdg}(N)$.

The extension class

$$
\kappa_{\mathrm{dR}}(\infty) \in \operatorname{Ext}_{\mathrm{MHS}}^{1}\left(H_{B}^{1}(X)^{\otimes 2}, H_{B}^{1}(X)\right),
$$

of $M_{\mathrm{B}}$ in the category of mixed Hodge structures arising from the exact sequence (16) is a natural object of study. We do so by considering, for each class $\xi \in \operatorname{Hdg}\left(H_{B}^{1}(X)^{\otimes 2}\right)$, the element

$$
\kappa_{\mathrm{dR}}(\xi ; \infty) \in \operatorname{Ext}_{\mathrm{MHS}}^{1}\left(\mathbb{Z}(-1), H_{B}^{1}(X)\right)
$$

3. A tantalising ongoing program (cf. [Ki]) also exploits the pro-unipotent fundamental group of a (hyperbolic, affine) curve $Y$ to bound its set of integral points, the rough idea being to associate to each point in $Y(\mathbb{Z})$ a system of non-abelian torsors, and then to control the Selmer varieties which parametrise them. 
associated to the class of the extension $M(\xi)=M(\xi ; \infty)$ appearing in the top row of the following commutative diagram with exact rows and cartesian squares:

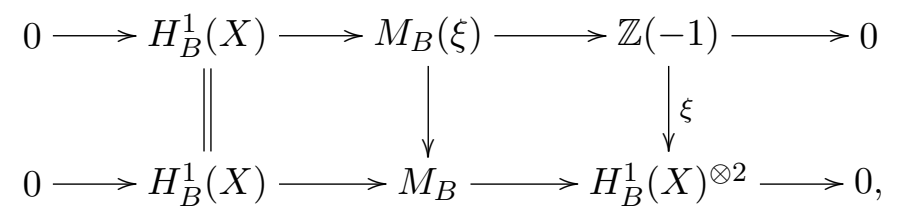

where the lower exact sequence is (16) applied to $Y$, once we identify $H_{B}^{1}(Y)=H_{B}^{1}(X)$ thanks to $(19)$.

There is a canonical isomorphism (cf. e.g. [Ca], [Hain3, §10.2])

$$
\operatorname{Ext}_{\mathrm{MHS}}^{1}\left(\mathbb{Z}(-1), H_{\mathrm{B}}^{1}(X)\right)=\frac{H_{\mathrm{dR}}^{1}(X / \mathbb{C})}{\Omega^{1}(X(\mathbb{C}))+H_{\mathrm{B}}^{1}(X)} .
$$

Recalling the exact sequence (17), this identification is obtained by choosing elements

$$
v_{\text {Hodge }} \in \operatorname{Fil}^{1}\left(M_{B}(\xi) \otimes \mathbb{C}\right)
$$

and $v_{\mathbb{Z}} \in M_{B}(\xi)$ satisfying

$$
\mathrm{p}\left(v_{\text {Hodge }}\right)=\mathrm{p}\left(v_{\mathbb{Z}}\right)=1,
$$

and assigning to the extension class $M_{B}(\xi)$ the element

$$
v_{\xi}:=i^{-1}\left(v_{\text {Hodge }}-v_{\mathbb{Z}}\right) \in H_{\mathrm{dR}}^{1}(X / \mathbb{C}) .
$$

Since $(38)$ determines $v_{\text {Hodge }}$ and $v_{\mathbb{Z}}$ up to elements of $\mathrm{Fil}^{1} H_{\mathrm{dR}}^{1}(X / \mathbb{C})=\Omega^{1}(X / \mathbb{C})$ and $H_{B}^{1}(X(\mathbb{C}), \mathbb{Z})$ respectively, the image of $v_{\xi}$ in the quotient on the right-hand side of $(37)$ depends only on the isomorphism class of the extension $M_{B}(\xi)$.

The Poincaré pairing

$$
H_{\mathrm{dR}}^{1}(X / F) \times H_{\mathrm{dR}}^{1}(X / F) \longrightarrow F
$$

with $F=\mathbb{C}$ induces an isomorphism

$$
\frac{H_{\mathrm{dR}}^{1}(X / \mathbb{C})}{\Omega^{1}(X(\mathbb{C}))+H_{\mathrm{B}}^{1}(X)} \longrightarrow \frac{\Omega^{1}(X(\mathbb{C}))^{\vee}}{H_{\mathrm{B}}^{1}(X)}=\operatorname{Jac}(X)(\mathbb{C}) .
$$

For all $\xi \in \operatorname{Hdg}\left(H_{B}^{1}(X)^{\otimes 2}\right)$, we continue to use $\kappa_{\mathrm{dR}}(\xi ; \infty) \in \operatorname{Jac}(X)(\mathbb{C})$ to $\operatorname{describe}$ the canonical point in the jacobian of $X$ arising from the identifications (37) and (41).

The assignment $\xi \mapsto \kappa_{\mathrm{dR}}(\xi ; \infty)$ therefore sets up a map

$$
\operatorname{Hdg}\left(H_{B}^{1}(X)^{\otimes 2}\right) \longrightarrow \operatorname{Jac}(X)(\mathbb{C})
$$

sending Hodge classes in $H_{\mathrm{dR}}^{1}(X)^{\otimes 2}$ to complex points in the Jacobian of $X$.

Lemma 1.3. - Given $\infty_{1}, \infty_{2} \in X(\mathbb{C})-\{o\}$, we have

$$
\kappa_{\mathrm{dR}}\left(\xi ; \infty_{1}\right)=\kappa_{\mathrm{dR}}\left(\xi ; \infty_{2}\right)+m_{\xi}\left(\left(\infty_{2}\right)-\left(\infty_{1}\right)\right)
$$

where we recall that $m_{\xi}=\int_{X(\mathbb{C})} \xi$ is the integral of any smooth two-form representing $\xi$ over the curve $X(\mathbb{C})$ diagonally embedded in $X(\mathbb{C}) \times X(\mathbb{C})$. 
Proof. — Let

$$
v_{\xi}\left(\infty_{1}\right) \in H_{\mathrm{dR}}^{1}\left(X-\left\{\infty_{1}\right\} / \mathbb{C}\right), \quad v_{\xi}\left(\infty_{2}\right) \in H_{\mathrm{dR}}^{1}\left(X-\left\{\infty_{2}\right\} / \mathbb{C}\right)
$$

be the elements attached to the extension classes $M_{B}(\xi)$ with $\infty=\infty_{1}$ and $\infty_{2}$ respectively, following equation (39). The difference $v_{\xi}\left(\infty_{1}\right)-v_{\xi}\left(\infty_{2}\right)$ corresponds, modulo elements of $\Omega^{1}(X)$ and $H_{B}^{1}(X(\mathbb{C}), \mathbb{Z})$, to the class in $H_{\mathrm{dR}}^{1}\left(X-\left\{\infty_{1}, \infty_{2}\right\}\right)$ of a regular differential $\omega \in$ $\Omega_{\log }^{1}\left(X-\left\{\infty_{1}, \infty_{2}\right\}\right)$ whose residual divisor is $m_{\xi}\left(\left(\infty_{2}\right)-\left(\infty_{1}\right)\right)$. But such an element describes the image of the degree zero divisor $m_{\xi}\left(\left(\infty_{2}\right)-\left(\infty_{1}\right)\right)$ in $\operatorname{Ext}^{1}\left(\mathbb{Z}(-1), H_{\mathrm{dR}}^{1}(X / \mathbb{C})\right)$, and the result follows.

It is occasionally convenient to attach an invariant $\kappa_{\mathrm{dR}}(\xi) \in \operatorname{Ext}^{1}\left(\mathbb{Z}(-1), H_{\mathrm{dR}}^{1}(X / \mathbb{C})\right)$ to an arbitrary element of $\operatorname{Hdg}\left(H_{B}^{1}(X)^{\otimes 2}\right)$ depending only on the pointed curve $(X ; o)$ and its fundamental group, and not on the choice of an auxiliary base point $\infty \in X-\{o\}$. We can do this by setting

$$
\kappa_{\mathrm{dR}}(\xi):=\kappa_{\mathrm{dR}}(\xi ; \infty)+m_{\xi}((\infty)-(o))
$$

By Lemma 1.3, the right hand side does not depend on the point $\infty$. It depends only on the Hodge class $\xi$ and the exact sequence (16) arising from the fundamental group $\pi_{1}(X ; o)$. It can be interpreted as arising from the pro-nilpotent fundamental group of $X-\{o\}$ with "tangential base point" at $o$, in the sense of the article [De].

The next two propositions express the points $\kappa_{\mathrm{dR}}(\xi ; \infty)$ and $\kappa_{\mathrm{dR}}(\xi)$ in terms of iterated integrals. Let us first introduce a few notations.

Given a Hodge class $\xi \in \operatorname{Hdg}\left(H_{B}^{1}(X)^{\otimes 2}\right)$, choose a representative

$$
\tilde{\xi}=\sum_{j=1}^{t} \omega_{j} \otimes \eta_{j}
$$

for its de Rham cohomology class, in such a way that $\omega_{j}$ and $\eta_{j}$ are smooth closed one-forms on $X$ for each $j$, at least one of which is of type $(1,0)$ (and hence, holomorphic on $X$ ). If $\rho \in \Omega^{1}(X)$ is any holomorphic differential, let $\gamma_{\rho} \in \mathbb{C}\left[\Gamma_{\text {ab }}\right]$ be a path whose homology class is Poincaré dual to $\rho$, i.e., such that

$$
\int_{\gamma_{\rho}} \tilde{\eta}=\langle\rho, \eta\rangle, \quad \text { for all } \eta \in H_{\mathrm{dR}}^{1}(X / \mathbb{C}) .
$$

Proposition 1.4. - With notations as above, let $\alpha_{\infty}$ be a smooth differential on $Y(\mathbb{C})$ of type $(1,0)$, with at worst logarithmic poles at $\infty$, satisfying

$$
d \alpha_{\infty}=\sum_{j=1}^{t} \omega_{j} \wedge \eta_{j} \text { on } Y(\mathbb{C})
$$


Then, for all $\rho \in \Omega^{1}(X / \mathbb{C})$,

$$
\begin{aligned}
\kappa_{\mathrm{dR}}(\xi ; \infty)(\rho) & =\int_{\gamma_{\rho}}\left(\sum_{j=1}^{t} \omega_{j} \cdot \eta_{j}-\alpha_{\infty}\right) \text { and } \\
\kappa_{\mathrm{dR}}(\xi)(\rho) & =\int_{\gamma_{\rho}}\left(\sum_{j=1}^{t} \omega_{j} \cdot \eta_{j}-\alpha_{\infty}\right)+m_{\xi} \int_{o}^{\infty} \rho .
\end{aligned}
$$

Proof. - The first equality is a direct consequence of the definitions, using the fact that the class of the extension $M_{B}(\xi ; \infty)$ attached to the pair $(\xi, \infty)$ is described by the iterated integral $J_{\tilde{\xi}, \alpha_{\infty}}$. The second equality then follows from equation (43) defining $\kappa_{\mathrm{dR}}(\xi)$.

We conclude this section with a formula analogous to the one in Proposition 1.4, expressing $\kappa_{\mathrm{dR}}(\xi ; \infty)$ and $\kappa_{\mathrm{dR}}(\xi)$ in terms of differentials of the second kind. This formula turns out to be particularly useful in computing the points $\kappa_{\mathrm{dR}}(\xi)$ numerically, as it is done in [DDLR] for Hodge classes arising from Hecke correspondences on a classical elliptic modular curve. Choose a representative

$$
\tilde{\xi}=\sum_{j=1}^{s} \omega_{j}^{+} \otimes \eta_{j}^{+}+\sum_{j=1}^{t} \eta_{j}^{-} \otimes \omega_{j}^{-} \in \Omega^{1}(X / \mathbb{C}) \otimes \Omega_{\mathrm{mer}}^{1}(X / \mathbb{C})+\Omega_{\mathrm{mer}}^{1}(X / \mathbb{C}) \otimes \Omega^{1}(X / \mathbb{C})
$$

of the class $\xi$, in such a way that the elements $\omega_{j}^{ \pm}$belong to $\Omega^{1}(X)=\operatorname{Fil}^{1} H_{\mathrm{dR}}^{1}(X / \mathbb{C})$ and the differentials $\eta_{j}^{ \pm}$are of the second kind and regular at $\infty$.

Proposition 1.5. - With notations as above, let $\alpha_{\infty}$ be a meromorphic differential on $X$, with at worst logarithmic poles at $\infty$, satisfying

$$
\underline{\mathrm{pp}}_{x}\left(\alpha_{\infty}\right)=\sum_{j=1}^{s} \underline{\mathrm{pp}}_{x}\left(\omega_{j}^{+} F_{\eta_{j}^{+}}\right)-\sum_{j=1}^{t} \underline{\mathrm{pp}}_{x}\left(\omega_{j}^{-} F_{\eta_{j}^{-}}\right), \quad \text { for all } x \in Y(\mathbb{C}) .
$$

Then, for all $\rho \in \Omega^{1}(X / \mathbb{C})$,

$$
\kappa_{\mathrm{dR}}(\xi ; \infty)(\rho)=\int_{\gamma_{\rho}}\left(\sum_{j=1}^{s} \omega_{j}^{+} \cdot \eta_{j}^{+}+\sum_{j=1}^{t} \eta_{j}^{-} \cdot \omega_{j}^{-}-\alpha_{\infty}\right) .
$$

Proof. - The proof is the same as for Proposition 1.4, using this time the algebraic description of the Hodge filtration on $M_{\mathrm{dR}, \mathbb{C}}$ in terms of differentials of the second kind.

\section{Points arising from algebraic cycles}

Hodge classes in $H_{\mathrm{B}}^{1}(X)^{\otimes 2}$ can be constructed naturally from geometry, by exploiting the cycle class maps

$$
\mathrm{cl}: \operatorname{Pic}(X \times X) \longrightarrow \operatorname{Hdg}\left(H_{B}^{2}(X \times X)\right) .
$$

More precisely, let $\varepsilon$ be the projector on $\operatorname{Pic}(X \times X)$ given by the rule

$$
\varepsilon(Z)=Z-i_{1, *} \pi_{1, *}(Z)-i_{2, *} \pi_{2, *}(Z),
$$


where

$$
\pi_{1}, \pi_{2}: X \times X \longrightarrow X, \quad \pi_{1}\left(P_{1}, P_{2}\right)=P_{1}, \quad \pi_{2}\left(P_{1}, P_{2}\right)=P_{2}
$$

are the natural projections onto the first and second factors, and

$$
i_{1}, i_{2}: X \hookrightarrow X \times X, \quad i_{1}(P)=(P, o), \quad i_{2}(P)=(o, P)
$$

are the natural inclusions of $X$ into the horizontal and vertical copies of $X$ in $X \times X$ over the point $o$.

The idempotent $\varepsilon$ annihilates the factors $H^{2}(X) \otimes H^{0}(X)$ and $H^{0}(X) \otimes H^{2}(X)$ in the Künneth decomposition of $H^{2}\left(X^{2}\right)$ (for any of the cohomology theories, Betti, de Rham or étale). For any $Z \in \operatorname{Pic}(X \times X)(F)$ we can thus define

$$
\xi_{Z}:=\operatorname{cl}(\varepsilon Z) \in \operatorname{Hdg}\left(H_{B}^{1}(X, \mathbb{Z})^{\otimes 2}\right) .
$$

In this way, we can assign to the cycle $Z \subset X \times X$ a point

$$
\kappa_{\mathrm{dR}}\left(\xi_{Z}\right) \in \operatorname{Jac}(X)(\mathbb{C})
$$

The goal of this section is to associate to any $Z \in \operatorname{Pic}(X \times X)(F)$ a point

$$
P_{Z} \in \operatorname{Jac}(X)(F)
$$

in such a way that for all complex embeddings $\iota: F \longrightarrow \mathbb{C}$,

$$
\kappa_{\mathrm{dR}}\left(\xi_{Z}\right)=P_{Z} \times_{\iota} \operatorname{Spec}(\mathbb{C}) .
$$

Let us describe a geometric recipe for constructing the desired point $P_{Z}$. Given a smooth projective variety $V_{/ F}$ and a positive integer $c \geq 1$, let

$$
\mathrm{CH}^{c}(V)=\mathcal{Z}^{c}(V) / \sim_{\text {rat }}
$$

denote the abelian group of rational equivalence classes of cycles of codimension $c$ in $V$. We regard it as a functor from the category of field extensions of $F$ in $\mathbb{C}$ to the category of abelian groups, given by the rule

$$
K / F \mapsto \mathrm{CH}^{c}(V)(K):=\left\{[Z]: Z \in \mathcal{Z}^{c}(V), \quad \text { such that }{ }^{\sigma} Z \sim_{\text {rat }} Z \text { for all } \sigma \in \operatorname{Aut}_{K}(\mathbb{C})\right\}
$$

For any integer $i \geq 1$, let $X_{i}=X$ be a copy of the curve $X$ and for any subset $\left\{i_{1}, \ldots, i_{m}\right\} \subseteq$ $\{1, \ldots, n\}$ with $m \leq n$ of indices in a given finite set, let $X_{i_{1}, \ldots, i_{m}}$ denote a copy of the curve $X$ embedded in $X_{1} \times \cdots \times X_{n}$ via the map

$$
x \mapsto\left(x_{i}\right)_{i=1}^{n}, \quad \text { where } x_{i}= \begin{cases}x & \text { if } i \in\left\{i_{1}, \ldots, i_{m}\right\}, \\ o & \text { otherwise. }\end{cases}
$$

The one-dimensional cycle $Z \subset X_{1} \times X_{2}$ gives rise to the following divisor classes on $X$ :

$$
D_{12}=Z \cdot X_{12}, \quad D_{1}=Z \cdot X_{1}, \quad D_{2}=Z \cdot X_{2} \text {. }
$$

Set

$$
P_{Z}=D_{12}-\operatorname{deg}\left(D_{12}\right) \cdot o \quad \in \quad \operatorname{Pic}^{0}(X)=\operatorname{Jac}(X)
$$

We now give an alternate geometric description of $P_{Z}$ which will be used to relate this point to iterated integrals. Let

$$
\Pi_{Z}:=Z_{12} \times X_{34} \subset X_{1} \times X_{2} \times X_{3} \times X_{4}
$$


where $Z_{12}$ denotes a copy of the cycle $Z$ embedded in $X_{1} \times X_{2}$. The class of the cycle $\Pi_{Z}$ belongs to $\mathrm{CH}^{2}\left(X_{1} \times X_{2} \times X_{3} \times X_{4}\right)$, and induces a morphism

$$
\Pi_{Z}: \mathrm{CH}^{2}\left(X_{1} \times X_{2} \times X_{3}\right) \longrightarrow \mathrm{CH}^{1}\left(X_{4}\right)
$$

by the usual rule

$$
\Pi_{Z}(\Delta)=\pi_{4 *}\left(\Pi_{Z} \cdot \pi_{123}^{*} \Delta\right)
$$

where

$$
\pi_{4}: X_{1} \times X_{2} \times X_{3} \times X_{4} \longrightarrow X_{4}, \quad \pi_{123}: X_{1} \times X_{2} \times X_{3} \times X_{4} \longrightarrow X_{1} \times X_{2} \times X_{3}
$$

denote the natural projections onto the fourth and first three factors respectively.

The Gross-Kudla-Schoen modified diagonal cycle in $X^{3}=X_{1} \times X_{2} \times X_{3}$ is defined to be

$$
\Delta_{\mathrm{GKS}}=X_{123}-X_{23}-X_{13}-X_{12}+X_{1}+X_{2}+X_{3} \in \mathrm{CH}^{2}\left(X_{1} \times X_{2} \times X_{3}\right) \text {. }
$$

Lemma 2.1. - For all $Z \in \operatorname{Pic}(X \times X)$,

$$
P_{Z}=\Pi_{Z}\left(\Delta_{\mathrm{GKS}}\right)
$$

Proof. - A direct calculation using only the definition (46) shows that

$$
\begin{gathered}
\Pi_{Z}\left(X_{123}\right)=D_{12}, \\
\Pi_{Z}\left(X_{23}\right)=D_{2}, \quad \Pi_{Z}\left(X_{13}\right)=D_{1}, \quad \Pi_{Z}\left(X_{12}\right)=\operatorname{deg}\left(D_{12}\right) \cdot o, \\
\Pi_{Z}\left(X_{1}\right)=\operatorname{deg}\left(D_{1}\right) \cdot o, \quad \Pi_{Z}\left(X_{2}\right)=\operatorname{deg}\left(D_{2}\right) \cdot o, \quad \Pi_{Z}\left(X_{3}\right)=0 .
\end{gathered}
$$

The lemma follows.

Remark 2.2. - Let $\infty$ be any point in $X$ (possibly equal to $o$ ). Viewing $X_{1} \times\{\infty\}$ and $\{\infty\} \times X_{2}$ as divisors in $X_{1} \times X_{2}$, a direct calculation shows that

$$
P_{X_{1} \times\{\infty\}}=P_{\{\infty\} \times X_{2}}=0 .
$$

Hence $P_{Z}=P_{\varepsilon Z}$ and the point $P_{Z}$ depends only on the Hodge class $\xi_{Z} \in \operatorname{Hdg}\left(H_{B}^{1}(X)^{\otimes 2}\right)$.

We will use Lemma 2.1 to derive an analytic formula for the point $P_{Z}$, or rather, for its image under the Abel-Jacobi map

$$
\mathrm{AJ}_{X}: \mathrm{CH}^{1}(X)_{0} \longrightarrow \frac{\Omega^{1}(X / \mathbb{C})^{\vee}}{H_{1}(X(\mathbb{C}), \mathbb{Z})} .
$$

This Abel-Jacobi map is defined on degree 0 divisors $D$ by the familiar rule:

$$
\operatorname{AJ}_{X}(D)(\omega)=\int_{\partial^{-1}(D)} \omega,
$$

where $\partial^{-1}(D)$ is any smooth one-chain on $X(\mathbb{C})$ whose boundary is $D$. It admits a higher dimensional generalisation

$$
\mathrm{AJ}_{X^{3}}: \mathrm{CH}^{2}\left(X^{3}\right)_{0}(\mathbb{C}) \longrightarrow J^{2}\left(X^{3}\right),
$$

where $\mathrm{CH}^{2}\left(X^{3}\right)_{0}$ is the Chow group of null-homologous cycles on $X^{3}$, and

$$
J^{2}\left(X^{3}\right)=\frac{\mathrm{Fil}^{2} H_{\mathrm{dR}}^{3}\left(X^{3} / \mathbb{C}\right)^{\vee}}{H_{3}\left(X^{3}(\mathbb{C}), \mathbb{Z}\right)}
$$


is the intermediate Jacobian associated to $H^{3}\left(X^{3}\right)$. This Abel-Jacobi map is defined by the rule generalising (48),

$$
\mathrm{AJ}_{X^{3}}(\Delta)(\omega)=\int_{\partial^{-1}(\Delta)} \omega, \quad \omega \in \mathrm{Fil}^{2} H_{\mathrm{dR}}^{3}\left(X^{3} / \mathbb{C}\right) .
$$

The following lemma verifies that the Gross-Kudla-Schoen cycle $\Delta_{\text {GKS }}$ lies in the domain of this Abel-Jacobi map.

Lemma 2.3. - The cycle $\Delta_{\mathrm{GKS}}$ is null-homologous.

Proof. - The obvious method to prove this lemma is to exploit the Künneth decomposition of the cohomology of $X_{1} \times X_{2} \times X_{3}$, as it is done in [GrSc] for example. We give a more detailed, direct argument, which yields a differentiable 3-chain on $X_{1} \times X_{2} \times X_{3}$ having $\Delta_{\text {GKS }}$ as boundary. While not strictly necessary to prove Lemma 2.3, such an explicit 3-chain will be used in subsequent calculations.

Let $g$ be the genus of the projective curve $X$, and let $\gamma_{1}, \ldots, \gamma_{2 g}$ be a collection of paths in $\mathbb{P}(X ; o)$ whose associated homology classes, denoted $\left[\gamma_{1}\right], \ldots,\left[\gamma_{2 g}\right]$, form an integral basis for $H_{1}(X(\mathbb{C}), \mathbb{Z})$. Let $\gamma_{1}^{*}, \ldots, \gamma_{2 g}^{*}$ be another collection of paths, whose homology classes give rise to the dual basis in $H_{1}(X(\mathbb{C}), \mathbb{Z})$ relative to the intersection product. In other words, we require that

$$
\left(\left[\gamma_{i}\right] \cdot\left[\gamma_{j}^{*}\right]\right)=\delta_{i j}, \quad \text { for all } 1 \leq i, j \leq 2 g,
$$

where $\delta_{i j}$ is the Kronecker delta. Let $\Delta$ denote the diagonal in $X \times X$. Its class in $H_{2}(X \times X)$ is given by

$$
\operatorname{cl}(\Delta)=\operatorname{cl}(X \times\{o\})+\operatorname{cl}(\{o\} \times X)+\sum_{i=1}^{2 g}\left[\gamma_{i}\right] \otimes\left[\gamma_{i}^{*}\right],
$$

and therefore there is a smooth 3-chain $W \subset X \times X$ such that

$$
\Delta=(X \times\{o\})+(\{o\} \times X)+\sum_{i=1}^{2 g} \gamma_{i} \times \gamma_{i}^{*}+\partial(W) .
$$

Applying this relation to $X_{123}$, viewed as the diagonal in $X_{12} \times X_{3}$, gives

$$
X_{123}=X_{12}+X_{3}+\sum_{i=1}^{2 g} \gamma_{i}(12) \times \gamma_{i}^{*}(3)+\partial\left(W_{12,3}\right),
$$

where

1. For $\{r, s\} \in\{1,2,3\}$, the regions $\gamma_{i}(r s)$ or $\gamma_{i}(r)$ are the paths $\gamma_{i}$ diagonally embedded in $X_{r s} \subset X_{1} \times X_{2} \times X_{3}$ and in $X_{r}$ respectively, and similar conventions are adopted for the paths $\gamma_{j}^{*}$.

2. The three-chain $W_{12,3}$ is a copy of the region $W$ of (51), embedded in $X_{12} \times X_{3}$ in the natural way.

By an analogous reasoning, we find

$$
X_{13}=X_{1}+X_{3}+\sum_{i=1}^{2 g} \gamma_{i}(1) \times \gamma_{i}^{*}(3)+\partial\left(W_{13}\right),
$$


and

$$
X_{23}=X_{2}+X_{3}+\sum_{i=1}^{2 g} \gamma_{i}(2) \times \gamma_{i}^{*}(3)+\partial\left(W_{23}\right)
$$

with the obvious meaning given to $W_{13} \subset X_{1} \times\{o\} \times X_{3}$ and to $W_{23} \subset\{o\} \times X_{2} \times X_{3}$. Subtracting (53) and (54) from (52), we obtain

$$
\Delta_{\mathrm{GKS}}=\sum_{i=1}^{2 g}\left(\gamma_{i}(12)-\gamma_{i}(1)-\gamma_{i}(2)\right) \times \gamma_{i}^{*}(3)+\partial\left(W_{12,3}-W_{13}-W_{23}\right) .
$$

But

$$
\gamma_{i}(12)-\gamma_{i}(1)-\gamma_{i}(2)=\partial\left(T_{\gamma_{i}}(12)\right)
$$

where

$$
T_{\gamma}(12)=\{(\gamma(r), \gamma(s)), \quad 0 \leq s \leq r \leq 1\} \subset X_{1} \times X_{2}
$$

is the two-dimensional region introduced in (25). Therefore,

$$
\Delta_{\mathrm{GKS}}=\partial\left(\sum_{i=1}^{2 g} T_{\gamma_{i}}(12) \times \gamma_{i}^{*}(3)+W_{12,3}-W_{13}-W_{23}\right),
$$

and the result follows.

Lemma 2.4. - For all $Z \in \mathrm{CH}^{1}(X \times X)$, and all $\rho \in \Omega^{1}(X)$,

$$
\operatorname{AJ}_{X}\left(P_{Z}\right)(\rho)=\operatorname{AJ}_{X^{3}}\left(\Delta_{\mathrm{GKS}}\right)\left(\xi_{Z}(12) \wedge \rho(3)\right),
$$

where the classes

$$
\xi_{Z}(12) \in \mathrm{Fil}^{1} H_{\mathrm{dR}}^{2}\left(X_{1} \times X_{2} / \mathbb{C}\right), \quad \rho(3) \in \Omega^{1}\left(X_{3}\right)=\mathrm{Fil}^{1} H_{\mathrm{dR}}^{1}\left(X_{3} / \mathbb{C}\right)
$$

are obtained in the natural way from the classes $\xi$ and $\rho$ respectively (so that, in particular, $\xi_{Z}(12) \wedge \rho(3)$ belongs to $\left.\mathrm{Fil}^{2} H_{\mathrm{dR}}^{3}\left(X^{3} / \mathbb{C}\right)\right)$.

Proof. - By Lemma 2.1 and the functoriality properties of the Abel-Jacobi map under correspondences,

$$
\operatorname{AJ}_{X}\left(P_{Z}\right)(\rho)=\operatorname{AJ}_{X}\left(\Pi_{Z} \Delta_{\mathrm{GKS}}\right)(\rho)=\mathrm{AJ}_{X^{3}}\left(\Delta_{\mathrm{GKS}}\right)\left(\Pi_{Z}^{*} \rho\right),
$$

where

$$
\Pi_{Z}^{*}: H_{\mathrm{dR}}^{1}\left(X_{4} / \mathbb{C}\right) \longrightarrow H_{\mathrm{dR}}^{3}\left(X_{1} \times X_{2} \times X_{3} / \mathbb{C}\right)
$$

is the morphism induced on de Rham cohomology by the correspondence $\Pi_{Z}=Z_{12} \times X_{34}$. A direct calculation reveals that

$$
\Pi_{Z}^{*}(\rho)=\operatorname{cl}(Z) \wedge \rho
$$

and therefore

$$
\mathrm{AJ}_{X}\left(P_{Z}\right)(\rho)=\mathrm{AJ}_{X^{3}}\left(\Delta_{\mathrm{GKS}}\right)(\operatorname{cl}(Z) \wedge \rho) .
$$

By a similar reasoning,

$$
\operatorname{AJ}_{X}\left(P_{\varepsilon Z}\right)(\rho)=\operatorname{AJ}_{X^{3}}\left(\Delta_{\mathrm{GKS}}\right)\left(\xi_{Z} \wedge \rho\right),
$$

and the result follows from Remark 2.2.

For the next proposition, we adopt the same notations that were introduced in the statement of Proposition 1.4 and the discussion immediately preceding it. 
Theorem 2.5. - For all $\xi \in \operatorname{Hdg}\left(H_{B}^{1}(X)^{\otimes 2}\right)$ and all $\rho \in \Omega^{1}(X / \mathbb{C})$,

$$
\operatorname{AJ}_{X^{3}}\left(\Delta_{\mathrm{GKS}}\right)(\xi(12) \wedge \rho(3))=\int_{\gamma_{\rho}}\left(\sum_{j=1}^{t} \omega_{j} \cdot \eta_{j}-\alpha_{\infty}\right)+m_{\xi} \int_{o}^{\infty} \rho .
$$

Proof. - By (55), and the definition of the Abel-Jacobi map,

$$
\mathrm{AJ}\left(\Delta_{\mathrm{GKS}}\right)(\xi \wedge \rho)=\sum_{i=1}^{2 g} \int_{T_{\gamma_{i}}(12) \times \gamma_{i}^{*}(3)} \tilde{\tilde{\xi}} \wedge \rho+\int_{W_{12,3}} \tilde{\xi} \wedge \rho
$$

where we have used the fact that $\tilde{\xi} \wedge \rho$ vanishes identically on $W_{13} \subset X_{1} \times\{o\} \times X_{3}$ and on $W_{23} \subset\{o\} \times X_{2} \times X_{3}$ to dispense with the integrals over these two regions. The first term appearing in the right hand side of (56) is equal to

$$
\sum_{i=1}^{2 g} \int_{T_{\gamma_{i}}(12) \times \gamma_{i}^{*}(3)} \tilde{\xi} \wedge \rho=\sum_{i=1}^{2 g} \int_{T_{\gamma_{i}}(12)} \tilde{\xi} \int_{\gamma_{i}^{*}(3)} \rho .
$$

This last expression can also be viewed as the integral of the two-form $\tilde{\xi}$ over the 2-chain

$$
\sum_{i=1}^{2 g}\left(\int_{\gamma_{i}^{*}} \rho\right) T_{\gamma_{i}}=T_{\gamma_{\rho}}
$$

where

$$
T_{\gamma_{\rho}}=\sum_{i=1}^{2 g} \lambda_{i} T_{\gamma_{i}}, \quad \text { with }\left[\gamma_{\rho}\right]=\sum \lambda_{i}\left[\gamma_{i}\right]
$$

By the formula for the iterated integral that is given in (25), it follows that

$$
\sum_{i=1}^{2 g} \int_{T_{\gamma_{i}}(12) \times \gamma_{i}^{*}(3)} \tilde{\xi} \wedge \rho=\int_{\gamma_{\rho}} \sum_{j=1}^{t} \omega_{j} \cdot \eta_{j} .
$$

To deal with the second term in the right-hand side of (56), we choose an auxiliary point $\infty$ which is not in the image of any of the paths $\gamma_{i}$ and $\gamma_{i}^{*}$, and such that $W_{12,3} \cap\left((\infty, \infty) \times X_{3}\right)$ is a smooth one-chain. By Lemma 1.2,

$$
\int_{W_{12,3}} \tilde{\xi} \wedge \rho=\int_{\partial W_{12,3}} \alpha_{\infty} \wedge \rho+m_{\xi} \int_{W_{12,3} \cap\left((\infty, \infty) \times X_{3}\right)} \rho .
$$

Recall that

$$
\partial W_{12,3}=X_{123}-X_{12}-X_{3}-\sum_{i=1}^{2 g} \gamma_{i}(12) \times \gamma_{i}^{*}(3),
$$

and note that the differential $\alpha_{\infty} \wedge \rho$ vanishes identically on $X_{123}, X_{12}$ and $X_{3}$ since it is of type $(2,0)$. Therefore, by a similar argument as above,

$$
\int_{\partial W_{12,3}} \alpha_{\infty} \wedge \rho=-\sum_{i=1}^{2 g} \int_{\gamma_{i}} \alpha_{\infty} \int_{\gamma_{i}^{*}} \rho=-\int_{\gamma_{\rho}} \alpha_{\infty} .
$$


Furthermore, the region of integration that appears in the second term of (58) has boundary

$$
\begin{aligned}
\partial\left(W_{12,3} \cap\left((\infty, \infty) \times X_{3}\right)\right) & =\left(X_{123}-X_{12}-X_{3}-\sum_{i=1}^{2 g} \gamma_{i} \times \gamma_{i}^{*}\right) \cap\left((\infty, \infty) \times X_{3}\right) \\
& =(\infty, \infty, \infty)-(\infty, \infty, o) .
\end{aligned}
$$

It follows that

$$
m_{\xi} \int_{W_{12,3} \cap\left((\infty, \infty) \times X_{3}\right)} \rho=m_{\xi} \int_{o}^{\infty} \rho .
$$

Substituting (59) and (60) into (58) yields

$$
\int_{W_{12,3}} \tilde{\xi} \wedge \rho=-\int_{\gamma_{\rho}} \alpha_{\infty}+m_{\xi} \int_{o}^{\infty} \rho .
$$

Finally, using (57) and (61) to rewrite the right hand side of (56), we obtain

$$
\operatorname{AJ}\left(\Delta_{\mathrm{GKS}}\right)(\xi \wedge \rho)=\int_{\gamma_{\rho}} \sum_{j=1}^{t} \omega_{j} \cdot \eta_{j}-\int_{\gamma_{\rho}} \alpha_{\infty}+m_{\xi} \int_{o}^{\infty} \rho,
$$

as was to be shown.

Corollary 2.6. - For all $Z \in \operatorname{Pic}(X \times X)$, and all $\rho \in \Omega^{1}(X)$,

$$
\operatorname{AJ}_{X}\left(P_{Z}\right)(\rho)=\int_{\gamma_{\rho}}\left(\sum_{j=1}^{t} \omega_{j} \cdot \eta_{j}-\alpha_{\infty}\right)+m_{\xi_{Z}} \int_{o}^{\infty} \rho
$$

where $\tilde{\xi}_{Z}=\sum_{i=1}^{t} \omega_{i} \otimes \eta_{i}$ is a representative of $\xi_{Z}:=\operatorname{cl}(\varepsilon Z)$ and $\alpha_{\infty}$ and $\gamma_{\rho}$ are as in the statement of Theorem 2.5.

Proof. — This follows directly from Lemma 2.4 and Theorem 2.5.

By combining Proposition 1.4 and Corollary 2.6 we obtain the sought-for relation between the extension classes $\kappa_{\mathrm{dR}}(\xi)$ arising from $\pi_{1}(X ; o)$ and the points $P_{Z}$.

Theorem 2.7. - For all $Z \in \operatorname{Pic}(X \times X), \kappa_{\mathrm{dR}}\left(\xi_{Z}\right)=P_{Z}$ in $\operatorname{Jac}(X)(\mathbb{C})$.

In light of Lemma 2.1, this is Theorem 1. As anticipated in the introduction, we can recover from it a result that is equivalent to the one obtained by Kaenders in $[\mathbf{K a}]$ :

Corollary 2.8. - Let $X_{12} \in \operatorname{Pic}(X \times X)$ be the class of the diagonal divisor in $X \times X$ up to rational equivalence. Then $\kappa_{\mathrm{dR}}\left(\xi_{X_{12}}\right)=(2 g-2) o-K$ in $\operatorname{Jac}(X)(\mathbb{C})$, where $K \in \operatorname{Pic}(X)$ is the canonical divisor on $X$.

Proof. - By Theorem 2.7 and $(45), \kappa_{\mathrm{dR}}\left(\xi_{X_{12}}\right)=P_{X_{12}}=X_{12} \cdot X_{12}-\operatorname{deg}\left(X_{12} \cdot X_{12}\right)$ o. An easy application of the Riemann-Roch theorem and the adjunction formula shows that the self-intersection of the diagonal in the square of a curve is $-K$. The corollary follows. 


\section{Modular and Shimura curves}

In this last section we specialize to the setting where $X$ is a Shimura curve over a totally real field.

Let $F$ be a totally real number field of degree $d$, and $R_{F}$ its ring of integers. Fix a classical Hilbert modular newform $g$ of parallel weight 2, level $N_{g}$ and trivial nebentypus. We refer the reader to $[\mathbf{Z h}]$ for the precise definitions of Shimura curves, Hecke correspondences and $L$-functions that we use throughout this section.

Choose a square-free ideal $D=\wp_{1} \cdot \ldots \cdot \wp_{r} \| N_{g}$ of $R_{F}$ dividing it exactly (by which we mean that $D$ and $N_{g} / D$ are relatively prime). Assume that $r+d$ is odd. (If $d$ is odd, a possible choice for $D$ is thus $D=R_{F}$ ).

Choose an ideal $N$ of $R_{F}$ such that $N_{g} \mid N$ and $D \| N$, and put $M=N / D$. Let $X_{0}^{D}(M)$ denote the Shimura curve associated with the Eichler order of level $M$ in a quaternion algebra ramified precisely at $D$ and at all archimedean places of $F$ but one, and write $J_{0}^{D}(M)$ for its jacobian.

Let $\mathbb{T}:=\left\langle T_{\wp}, \wp \nmid N\right\rangle_{\mathbb{Q}}$ be the Hecke algebra generated by the set of Hecke operators at primes $\wp$ not dividing $N$, acting on $J_{0}^{D}(M)$. This $\mathbb{Q}$-algebra is isomorphic to a free product of number fields, namely

$$
\mathbb{T} \simeq \prod_{D \| n \mid N} \prod_{h} K_{h}
$$

where for each ideal $n$ such that $D \| n$ and $n \mid N, h$ runs through a set of representatives for the orbits of $\operatorname{Gal}(\overline{\mathbb{Q}} / \mathbb{Q})$ acting on the set of Hilbert newforms of level $n$, and $K_{h}$ is the number field generated by the eigenvalues of $\mathbb{T}$ acting on $h$. In particular $\mathbb{T}$ contains a copy of $K_{g}$; write $T_{g} \in \mathbb{T}$ for the corresponding idempotent in the decomposition (63).

If $M$ is prime then in fact $\mathbb{T} \simeq$ End $J_{0}^{D}(M)$. But as soon as $M$ is a composite ideal, the endomorphism algebra of $J_{0}^{D}(M)$ is larger, namely a product of matrix algebras:

$$
\operatorname{End}^{0}\left(J_{0}^{D}(M)\right):=\operatorname{End}\left(J_{0}^{D}(M)\right) \otimes \mathbb{Q} \simeq \prod_{D \| n \mid N} \prod_{h} M_{\sigma(N / n)}\left(K_{h}\right),
$$

where $\sigma(N / n)$ is the number of ideal dividing $N / n$. Its center is thus $\mathbb{T}$.

Note that $\operatorname{End}^{0}\left(J_{0}^{D}(M)\right)[g]:=T_{g} \cdot \operatorname{End}^{0}\left(J_{0}^{D}(M)\right) \simeq M_{\sigma\left(N / N_{g}\right)}\left(K_{g}\right)$, the center of which is $\mathbb{T}[g]:=T_{g} \cdot \mathbb{T}$.

Since

$$
\operatorname{End}\left(J_{0}^{D}(M)\right) \simeq \frac{\operatorname{Pic}\left(X_{0}^{D}(M) \times X_{0}^{D}(M)\right)}{\pi_{1}^{*}\left(\operatorname{Pic}\left(X_{0}^{D}(M)\right)+\pi_{2}^{*}\left(\operatorname{Pic}\left(X_{0}^{D}(M)\right)\right.\right.}
$$

by [LaBi, Theorem 11.5.1], we can define

$$
H_{g}:=\left\{\xi_{T}, T \in \mathbb{T}[g]\right\} \subseteq \operatorname{Hdg}\left(H^{1}\left(X_{0}^{D}(M)\right)^{\otimes 2} \otimes \mathbb{Q}\right)
$$

to be the module of Hodge classes associated with $g$.

Definition 3.1. $-\underline{P}_{g}:=\kappa_{\mathrm{dR}}\left(H_{g}\right) \subseteq \mathbb{Q} \otimes J_{D}^{0}(M)(F)$.

The Hodge classes in $H_{g}$ can be written down explicitly. For instance, a representative for $\xi_{g}:=\xi_{T_{g}} \in H_{g}$ is given as follows: let $\left\{\omega_{g, i}\right\}$ be a basis of the $g$-isotypical component of 
the module of regular differential forms on $X_{0}^{D}(M)$ and complete it to a symplectic basis $\left\{\omega_{g, i}, \eta_{g, i}\right\}$ of $H_{\mathrm{dR}}^{1}\left(X_{0}^{D}(M)\right)[g]$. Then

$$
\tilde{\xi}_{g}=\sum_{i} \omega_{g, i} \otimes \eta_{g, i}-\eta_{g, i} \otimes \omega_{g, i}
$$

is a differential form on $X_{0}^{D}(M) \times X_{0}^{D}(M)$, rational over $F$, which represents $\xi_{g}$. The point $P_{g}:=\kappa_{\mathrm{dR}}\left(\xi_{g}\right) \in \underline{P}_{g}$ can then be computed as the tuple of periods of the iterated integral associated with (67); similar formulas can be obtained of course for a complete basis of $\underline{P}_{g}$ : see [DDLR] for explicit calculations.

Recall that $\kappa_{\mathrm{dR}}(\xi)$ depends on the choice of the base point $o$. If we are dealing with the classical elliptic modular curve associated to the split algebra $\mathrm{M}_{2}(\mathbb{Q})$, we can take $o$ to be the cusp at infinity, which is a rational point on $X_{0}(M)$.

If $X_{0}^{D}(M)$ is the Shimura curve attached to an order in a division quaternion algebra over an arbitrary totally real number field $F$, then $X_{0}^{D}(M)(F)$ may be empty: see e.g. [Sh75, Theorem 5.1], which establishes criteria for the existence of real points on these curves and shows in particular that $X_{0}^{D}(M)(\mathbb{R})=\emptyset$ when $F=\mathbb{Q}$. Instead of fixing a single point $o$ rational over $F$, one proceeds as in $[\mathbf{G r K u}, \S 13],[\mathbf{Y Z Z}]$ and $[\mathbf{Z h}]$ by rather considering a canonical Gal $(\bar{F} / F)$-invariant divisor class $o=\sum a_{i} o_{i}$ of degree 1 with rational coefficients on $X_{0}^{D}(M)$, which in $[\mathbf{Z h}]$ is called the Hodge class of $X_{0}^{D}(M)$. In this case, by $\kappa_{\mathrm{dR}}(\xi)$ we mean $\sum a_{i} \kappa_{\mathrm{dR}}\left(\xi ; o_{i}\right)$.

Choose now another normalised Hilbert newform $f$ of weight 2, level $N_{f} \mid N$ and trivial nebentypus, different from $g$. The triple product $L$-function $L(g \otimes g \otimes f, s)$, which was first studied by Garrett in [Gar], is the $L$-function associated with the tensor product $V_{g} \otimes V_{g} \otimes V_{f}$, where $V_{g}$ and $V_{f}$ denote the two-dimensional $\ell$-adic representations of $G_{F}:=\operatorname{Gal}(\bar{F} / F)$ attached to $g$ and $f$ respectively. It is explicitly given by an Euler product with Euler factors of degree 8 at the primes $\wp \nmid N$, and factors as

$$
L(g \otimes g \otimes f, s)=L(f, s-1) L\left(\mathrm{Sym}^{2} g \otimes f, s\right),
$$

mirroring the decomposition

$$
V_{g} \otimes V_{g}=\mathbb{Q}_{\ell}(-1) \oplus \operatorname{Sym}^{2} V_{g}
$$

of $\ell$-adic representations of $G_{F}$.

Thanks to the work of Garrett, Piatetski-Shapiro-Rallis, Harris-Kudla, Prasad and others (cf. [Gar], [Pr] and the references therein), it is known that $L(g \otimes g \otimes f, s)$ admits analytic continuation to $\mathbb{C}$ and satisfies a functional equation relating its values at $s$ and $4-s$. The sign $\epsilon$ in this functional equation is expressed as a product

$$
\epsilon=(-1)^{d} \prod_{\wp \mid \operatorname{gcd}\left(N_{g}, N_{f}\right)} \epsilon_{\wp}
$$

of local signs $\epsilon_{\wp} \in\{ \pm 1\}$; for primes $\wp$ dividing exactly both $N_{g}$ and $N_{f}$,

$$
\epsilon_{\wp}=-a_{\wp}(f)
$$

is the negative of the eigenvalue of the Hecke operator $T_{\wp}$ acting on $f$ (cf. $[\mathbf{G r K u}],[\mathbf{P r}]$ ), and in particular does not depend on the choice of $g$. When $\wp^{2} \mid N_{g}$ or $N_{f}$, the value of $\epsilon_{\wp}$ depends 
on the local behavior of the automorphic representations associated with $g$ and $f$; see $[\mathbf{P r}]$ for more details. Assume that

$$
\epsilon(f)=-1, \quad \epsilon\left(\operatorname{Sym}^{2}(g) \otimes f\right)=+1 .
$$

Then $\epsilon=-1$ and we can set $D=D_{g ; f}=\prod_{\epsilon_{\wp}=-1} \wp$; note that $D$ is the product of $r=\sharp\left\{\epsilon_{\wp}=-1\right\}$ distinct prime ideals, and that $r+d$ is odd by (69). Assume that $D \| N$ and put as above $M=M_{g ; f}=N / D$.

The quotient of $J_{0}^{D}(M)$ associated to $f$ by the theory of Eichler-Shimura is an abelian variety which is well-defined only up to $F$-isogenies, and is isogenous to the power $A_{f}^{\sigma\left(N / N_{f}\right)}$ of an $F$-simple abelian variety $A_{f}$. This means that there are $\sigma\left(N / N_{f}\right) F$-linear independent modular parametrizations

$$
\pi_{f}^{d}: J_{0}^{D}(M) \longrightarrow A_{f}
$$

of $A_{f}$, one for each divisor $d$ of $N / N_{f}$.

Definition 3.2. - Let

$$
\underline{P}_{g, f}:=\sum_{d \mid \frac{N}{N_{f}}} \pi_{f}^{d}\left(\underline{P}_{g}\right) \subseteq A_{f}(F) \otimes \mathbb{Q}
$$

be the subspace of the Mordell-Weil group (tensored with $\mathbb{Q}$ ) of $A_{f}$ associated to the pair of modular forms $(g, f)$.

In particular, if we let $\pi_{f}: J_{D}^{0}(M) \rightarrow J_{0}^{D}\left(N_{f} / D\right) \rightarrow A_{f}$ denote the map which factors through the natural projection of $J_{0}^{D}(M)$ onto $J_{0}^{D}\left(N / N_{f}\right)$, the point

$$
P_{g, f}=\pi_{f}\left(P_{g}\right)
$$

belongs to this space, and can be explicitly computed by means of (67).

Formula (72), combined with Theorem 2.5, provides a suggestive construction of a point on a modular abelian variety by means of iterated integrals. Theorem 1 can be used to show that, in the particular case that $g$ has rational fourier coefficients, $P_{g, f}$ is essentially equal to a point that was first introduced by $\mathrm{S}$. Zhang by a different method, whose definition we recall here for the convenience of the reader:

Definition 3.3. - [S. Zhang] Assume $g$ has fourier coefficients in $\mathbb{Z}$. Let $E_{g} / F$ be the elliptic curve corresponding to $g$ by the Eichler-Shimura construction and $\pi_{g}: X_{0}^{D}(M) \longrightarrow E_{g}$ be a modular parametrization of curves. Letting $O \in E_{g}(F)$ denote the identity element, define

$$
P_{g, f}^{Z h}:=\pi_{f, *} \pi_{g}^{*}(O) \text {. }
$$

We refer the reader to the forthcoming Ph. D thesis [Daub] of M. Daub for more details concerning the connection between (72) and Definition 3.3. See also W. Stein's appendix to [DDLR] for the effective numerical computation of the points $P_{g, f}^{Z h}$ in the classical setting of elliptic modular curves over $F=\mathbb{Q}$. Note that Definition 3.3 does not readily generalize to the case in which the fourier coefficients of $g$ are not integral, as the resulting modular abelian variety has dimension greater than 1 . On the other hand, formula (72) is available in that broader setting: see again [DDLR] for explicit examples. 
The algebra $\mathbb{T}$ of Hecke operators acts by correspondences on $\mathrm{CH}^{1}\left(X_{0}^{D}(M)\right) \otimes \mathbb{Q}$ and gives rise via $(65)$ to a linear action of $\mathbb{T} \otimes \mathbb{T} \otimes \mathbb{T}$ on $\mathrm{CH}^{2}\left(X_{0}^{D}(M)^{3}\right) \otimes \mathbb{Q}$ and on its subspace $\mathrm{CH}^{2}\left(X_{0}^{D}(M)^{3}\right)_{0} \otimes \mathbb{Q}$ of null-homologous cycles.

Definition 3.4. - Set

$$
\operatorname{MW}(g, f):=\operatorname{Hom}\left(\mathbb{T}[g] \otimes \mathbb{T}[g] \otimes \mathbb{T}[f], \mathrm{CH}^{2}\left(X_{0}^{D}(M)^{3}\right)_{0} \otimes \mathbb{Q}\right)
$$

and let $y_{g, f} \in \mathrm{MW}(g, f)$ be the homomorphism defined by

$$
y_{g, f}\left(T_{1} \otimes T_{2} \otimes T_{3}\right):=\left(T_{1} \otimes T_{2} \otimes T_{3}\right)\left(\Delta_{\mathrm{GKS}}\right) .
$$

By working with a suitable integral model of $X_{0}^{D}(M)^{3}$, B. Gross and C. Schoen have shown in $[\mathbf{G r S c}]$ that Beilinson-Bloch's $[\mathbf{B 1 8 4}]$ height pairing $\langle,\rangle^{\mathrm{BB}}$ on $\mathrm{CH}^{2}\left(X_{0}^{D}(M)^{3}\right)_{0}$ gives rise to a well-defined pairing

$$
\langle,\rangle^{\mathrm{BB}}: \mathrm{MW}(g, f) \times \operatorname{MW}(g, f) \longrightarrow \operatorname{Hom}\left((\mathbb{T}[g] \otimes \mathbb{T}[g] \otimes \mathbb{T}[f])^{\otimes 2}, \mathbb{C}\right),
$$

independently of any choices; see also [YZZ, §1.3].

X. Yuan, S. Zhang and W. Zhang have recently proved in [YZZ] the following formula, which was conjectured by B. Gross and S. Kudla in [GrKu, Conjecture 13.2]:

Theorem 3.5. - [YZZ, Theorem 1.3.1, Corollary 1.4.1 (1)] Assume as in (70) that $\epsilon(f)=-1$ and $\epsilon\left(\operatorname{Sym}^{2}(g) \otimes f\right)=+1$. Then

$$
\left\langle y_{g, f}, y_{g, f}\right\rangle^{\mathrm{BB}}=L^{\prime}(f, 1) \cdot \prod_{\sigma: K_{g} \rightarrow \mathbb{C}} L\left(g^{\sigma} \otimes g^{\sigma} \otimes f, 2\right) \cdot \lambda
$$

for some non-zero homomorphism $\lambda \in \operatorname{Hom}\left((\mathbb{T}[g] \otimes \mathbb{T}[g] \otimes \mathbb{T}[f])^{\otimes 2}, \mathbb{C}\right)$.

Remark 3.6. - Note the different language in which the results are presented in [YZZ] in contrast to [GrSc, §8] and here: while the former exploits the language of automorphic representations, the latter adopt the more classical parlance of modular forms and Hecke operators, working with Shimura curves of fixed finite level.

The combination of Theorem 1 together with Theorem 3.5 yields the following result.

Theorem 3.7. - Assume as in (70) that $\epsilon(f)=-1$ and $\epsilon\left(\operatorname{Sym}^{2}(g) \otimes f\right)=+1$. Then the space $\underline{P}_{g, f} \subseteq A_{f}(F) \otimes \mathbb{Q}$ has positive rank if and only if

$$
L^{\prime}(f, 1) \neq 0, \quad \text { and } \quad L\left(\operatorname{Sym}^{2}\left(g^{\sigma}\right) \otimes f, 2\right) \neq 0 \text { for all } \sigma: K_{g} \rightarrow \mathbb{C} .
$$

Proof. - The automorphism $\tau: X_{0}^{D}(M)^{3} \rightarrow X_{0}^{D}(M)^{3},\left(x_{1}, x_{2}, x_{3}\right) \mapsto\left(x_{2}, x_{1}, x_{3}\right)$ gives rise to an involution $\tau^{*}$ on $\mathrm{CH}^{2}\left(X_{0}^{D}(M)^{3}\right)_{0} \otimes \mathbb{Q}$ and on $J^{2}\left(X_{0}^{D}(M)^{3}\right)$ which preserves the $(g, g, f)$ isotypical components. By [YZZ, Corollary 1.4.1 (1)], the homomorphism $y_{g, f}$ is invariant under the action of $\tau$.

Hence, combined with Theorem 3.5, condition (73) holds if and only if there exist correspondences $T_{g, i} \in \mathbb{T}[g], T_{f, i} \in \mathbb{T}[f]$ such that,

$$
\langle\Delta[\breve{g}, \breve{g}, \breve{f}], \Delta[\breve{g}, \breve{g}, \breve{f}]\rangle^{\mathrm{BB}} \neq 0,
$$

where we set

$$
\Delta[\breve{g}, \breve{g}, \breve{f}]:=\sum_{i} T_{g, i} \otimes T_{g, i} \otimes T_{f, i}\left(\Delta_{\mathrm{GKS}}\right)
$$


Lemma 2.1 combined with Lemma 3.8 below show that

$$
\sum_{i} \Pi_{X_{12}} \cdot\left(T_{g, i} \otimes T_{g, i} \otimes 1\right)\left(\Delta_{\mathrm{GKS}}\right)=\sum_{i} \Pi_{T_{g, i}}\left(\Delta_{\mathrm{GKS}}\right) .
$$

Since $T \circ \Pi_{X_{12}}=\Pi_{X_{12}}(1 \otimes 1 \otimes T)$ for any $T \in \mathrm{CH}^{1}\left(X_{0}^{D}(M)^{2}\right)$, we obtain that

$$
\sum T_{f, i} \Pi_{T_{g, i}}\left(\Delta_{\mathrm{GKS}}\right)=\Pi_{X_{12}}(\Delta[\breve{g}, \breve{g}, \breve{f}]) .
$$

For each $i, T_{f, i}$ is a self-correspondence on $X_{0}^{D}(M)$ that gives rise to an endomorphism $\pi_{f, i}$ of $\operatorname{Jac}\left(X_{0}^{D}(M)\right)$ which is a linear combination of the projections $\pi_{f}^{d}, d \mid N / N_{f}$. Set

$$
\left.P[\breve{g}, \breve{g}, \breve{f}]:=\sum_{i} \pi_{f, i} \Pi_{T_{g, i}}\left(\Delta_{\mathrm{GKS}}\right)\right] \in A_{f}
$$

and write $\langle,\rangle^{N T}$ for the Néron-Tate canonical height pairing on an abelian variety. By [Bl84, p. 120-121, A.2], the pairing $\langle,\rangle^{\mathrm{BB}}$ is functorial with respect to correspondences, and from (74) it follows that

$$
\langle\Delta[\breve{g}, \breve{g}, \breve{f}], \Delta[\breve{g}, \breve{g}, \breve{f}]\rangle^{\mathrm{BB}} \doteq\langle P[\breve{g}, \breve{g}, \breve{f}], P[\breve{g}, \breve{g}, \breve{f}]\rangle^{N T}
$$

up to a positive constant.

Note that, by $(71), P[\breve{g}, \breve{g}, \breve{f}]$ is a point in $\underline{P}_{g, f}$. By Theorem 1 we have $P[\breve{g}, \breve{g}, \breve{f}]=$ $\sum_{i} \pi_{f, i} \kappa_{\mathrm{dR}}\left(\xi_{T_{g, i}}\right)$. The corollary follows.

Lemma 3.8. - Given a self-correspondence $Z \in \mathrm{CH}^{1}\left(X_{0}^{D}(M)^{2}\right)$ of $X_{0}^{D}(M)$, it holds that

$$
\Pi_{X_{12}} \cdot(Z \otimes Z \otimes 1)\left(\Delta_{\mathrm{GKS}}\right)=Z \cdot X_{12}-\operatorname{deg}\left(Z \cdot X_{12}\right) \text { o. }
$$

Proof. - By applying (46), it directly follows that $\Pi_{X_{12}} \cdot(Z \otimes Z \otimes 1)\left(X_{123}\right)=Z \cdot X_{12} \in$ $\operatorname{Jac}\left(X_{0}^{D}(M)\right)$. Similarly, when one applies (46) to the six other components $X_{j}, X_{j k}$ of $\Delta_{\text {GKS }}$ one obtains that, for all $j, k=1,2,3, j \neq k$, the divisors $\Pi_{X_{12}} \cdot(Z \otimes Z \otimes 1)\left(X_{j}\right)$ and $\Pi_{X_{12}} \cdot(Z \otimes Z \otimes 1)\left(X_{j k}\right)$ are all linearly equivalent to some multiple of the degree 1 divisor $o$. Since $\Delta_{\mathrm{GKS}}$ is null-homologous, $\Pi_{X_{12}} \cdot(Z \otimes Z \otimes 1)\left(\Delta_{\mathrm{GKS}}\right)$ is a divisor class on $X_{0}^{D}(M)$ of degree 0 . The lemma follows.

In light of Theorem 1, Theorem 3.7 can be viewed to some extent as an analogue for modular and Shimura curves of the Deligne-Wojtkowiak calculations [De] in which values of the Riemann zeta-function $\zeta(s)$ at odd positive integers emerge from the study of the mixed Tate motives arising in the pro-unipotent fundamental group of $\mathbb{P}_{1}-\{0,1, \infty\}$. It was motivated by the authors' study of the Chow-Heegner points attached to diagonal cycles in triple products of Kuga-Sato-varieties, which is detailed more systematically in [DR1]. Note however that while [De] obtains formulae for the values of $\zeta(s)$ at certain non-critical points, Theorem 3.7 applies only to the critical value of the $L$-function $L(g \otimes g \otimes f, s)$ at $s=2$.

\section{References}

[Bl77] S. Bloch, Applications of the dilogarithm function in algebraic K-theory and algebraic geometry, Proc. Int. Symp. Algebraic Geometry (Kyoto Univ., 1977), 103-114.

[Bl84] S. Bloch, Height pairings for algebraic cycles, J. Pure Appl. Algebra 34 (1984), 119-145. 
[BK] S. Bloch, K. Kato, L-functions and Tamagawa numbers of motives, in The Grothendieck Festschrift I, Progr. Math. 108, 333-400 (1993), Birkhauser.

[Ca] J. A. Carlson, Extensions of mixed Hodge structures, Journées de Géometrie Algébrique d'Angers, Juillet 1979 in Algebraic Geometry, Angers, (1979) 107-127.

[CCM] J. Carlson, C. H. Clemmens, J. Morgan, On the mixed Hodge structure associated to $\pi_{3}$ of a simply connected complex projective manifold, Ann. Sci. École Norm. Sup. 14:4 (1981), 323-338.

[Chen] K.-T. Chen, Iterated path integrals, Bull. Amer. Math. Soc. 83 (1977), 831-879.

[CG] E. Colombo, van Geemen, Note on curves on a jacobian, Compositio Math. 88 (1993), 333-353.

[DDLR] H. Darmon, M. Daub, S. Lichtenstein, V. Rotger, Algorithms for Chow-Heegner points via iterated integrals, with an appendix by W. Stein, submitted for publication.

[DP] H. Darmon and R. Pollack, The efficient calculation of Stark-Heegner points via overconvergent modular symbols, Israel Journal of Mathematics, 153 (2006), 319-354.

[DR1] H. Darmon, V. Rotger, Diagonal cycles and Euler systems I: a p-adic Gross-Zagier formula, submitted.

[Daub] M. Daub, Ph. D thesis, Berkeley, in progress.

[De] P. Deligne, Le groupe fondamental de la droite projective moins trois points, in Galois groups over $\mathbb{Q}$ (Berkeley, CA, 1987), 79-97, Math. Sci. Res. Inst. Publ. 16, Springer, New York, 1989.

[Dr] V. Drinfeld, On quasitriangular quasi-Hopf algebras and on a group that is closely connected with $\operatorname{Gal}(\overline{\mathbb{Q}} / \mathbb{Q})$, Algebra i Analiz 2 (1990), 149-181; translation in Leningrad Math. J. 2 (1991), 829-860.

[Gar] P. Garrett, Decomposition of Eisenstein series: Rankin triple products, Ann. Math. 125 (1987), 209-235.

[GrKu] B. Gross, S. Kudla, Heights and the central critical values of triple product L-functions, Compositio Math. 81 (1992), no. 2, 143-209.

[GrSc] B. Gross, C. Schoen, The modified diagonal cycle on the triple product of a pointed curve, Ann. Inst. Fourier (Grenoble) 45 (1995), no. 3, 649-679.

[GrZa] B. Gross, D. Zagier, Heegner points and derivatives of L-series, Invent. Math. 84 (1986), $225-320$.

[Hain1] R. Hain, The de Rham homotopy theory of complex algebraic varieties, I and II, K-Theory 1 (1987), 271-324 and 481-497.

[Hain2] R. Hain, The geometry of the mixed Hodge structure on the fundamental group, (Bowdoin, 1985), Proc. Sympos. Pure Math. 46:2, Amer. Math. Soc., Providence, RI, 1987, 247-82.

[Hain3] R. Hain, Iterated integrals and algebraic cycles: examples and prospects, in Cont. Trends in Alg. Geometry and Alg. Topology, S. Chern, L. Fu, R. Hain (ed.), Nankai Tracts in Math. 5, Singapore, 2002.

[Har] B. Harris, Chen's iterated integrals and algebraic cycles, in Cont. Trends in Alg. Geometry and Alg. Topology, S. Chern, L. Fu, R. Hain (ed.), Nankai Tracts in Math. 5, Singapore, 2002.

[Ka] R. H. Kaenders, The mixed Hodge structure of the fundamental group of a punctured Riemann surface, Proc. Amer. Math. Soc. 129 (2001), 1271-1281.

[Ki] M. Kim, $p$-adic $L$-functions and Selmer varieties associated to elliptic curves with complex multiplication, Ann. Math. (2) 172 (2010), 751-759.

[La] S. Lang, Introduction to Arakelov Theory, Springer, 1988.

[LaBi] H. Lange, Ch. Birkenhake, Complex abelian varieties. Grundlehren der mathematischen Wissenschaften 302, Springer-Verlag (1992). 
[Ne] J. Nekovář, Kolyvagin's method for Chow groups of Kuga-Sato varieties, Invent. Math. 107 (1992), 99-125.

[Pr] D. Prasad, Relating invariant linear form and local epsilon factors via global methods, with an appendix by H. Saito, Duke J. Math. 138:2 (2007), 233-261.

$[\mathrm{Pu}] \mathrm{M}$. Pulte, The fundamental group of a Riemann surface: mixed Hodge structures and algebraic cycles, Duke Math. J. 57 (1988), 721-760.

[Sh75] G. Shimura, On the real points of an arithmetic quotient of a bounded symmetric domain, Math. Annalen 215 (1975), 135-164.

[YZZ] X. Yuan, S. Zhang, W. Zhang, Triple product L-series and Gross-Schoen cycles I: split case, preprint.

[Zh] S. Zhang, Heights of Heegner points on Shimura curves, Ann. Math. (2) 153:1 (2001), 27-147.

[Zh2] S. Zhang, Arithmetic of Shimura curves, Science China Mathematics, 2010, Volume 53, Number 3, Pages 573-592.

September 13, 2012

Henri Darmon, H. D.: Montreal, Department of Mathematics, McGill University, Montreal, Canada E-mail : henri.darmon@mcgill.ca

Victor Rotger, V. R.: Dep. Matemàtica Aplicada II, Universitat Politècnica de Catalunya, Barcelona, Spain - E-mail : victor.rotger@upc.edu

Ignacio Sols, I. S.: Departamento de Álgebra, Universidad Complutense de Madrid, Spain

E-mail : isols@mat.ucm.es 